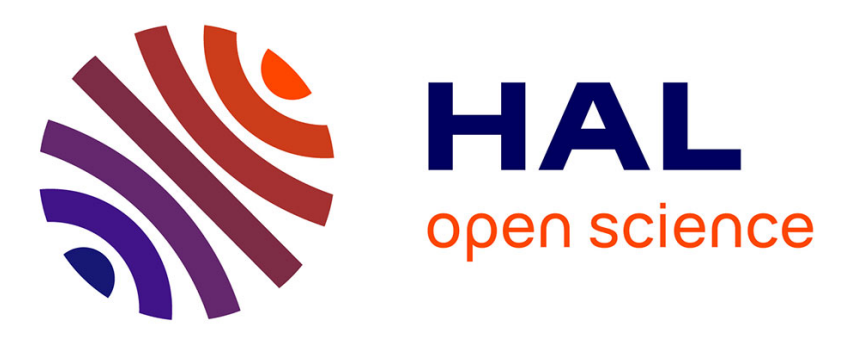

\title{
A Lattice Boltzmann Model for the Simulation of Flows in Open Channels with Application to Flows in a Submerged Sluice Gate
}

Olivier Marcou, Bastien Chopard, Samira El Yacoubi, Boussad Hamroun, Laurent Lefevre, Eduardo Mendes

\section{To cite this version:}

Olivier Marcou, Bastien Chopard, Samira El Yacoubi, Boussad Hamroun, Laurent Lefevre, et al.. A Lattice Boltzmann Model for the Simulation of Flows in Open Channels with Application to Flows in a Submerged Sluice Gate. Journal of Irrigation and Drainage Engineering, 2010, 136 (12), pp.809 - 822. 10.1061/(ASCE)IR.1943-4774.0000260 . hal-01625050

\section{HAL Id: hal-01625050 https://hal.science/hal-01625050}

Submitted on 27 Oct 2017

HAL is a multi-disciplinary open access archive for the deposit and dissemination of scientific research documents, whether they are published or not. The documents may come from teaching and research institutions in France or abroad, or from public or private research centers.
L'archive ouverte pluridisciplinaire HAL, est destinée au dépôt et à la diffusion de documents scientifiques de niveau recherche, publiés ou non, émanant des établissements d'enseignement et de recherche français ou étrangers, des laboratoires publics ou privés. 


\title{
A Lattice Boltzmann Model for the Simulation of
}

Flows in Open Channels with Application to Flows

\author{
in a submerged sluice gate
}
O. Marcou*
B. Chopard ${ }^{\dagger}$
S. El Yacoubi ${ }^{\ddagger}$
B. Hamroun ${ }^{\S}$
L. Lefèvre
E. Mendes ${ }^{\|}$

*PhD, Laboratoire de Mathématiques, Physique et Systèmes (LAMPS), Université de Perpignan Via Domitia (UPVD),Perpignan, France and Computer Science Department, University of Geneva, Switzerland, marcou@univ-perp.fr

${ }^{\dagger}$ Professor, Computer Science Department,University of Geneva, Switzerland, Bastien.Chopard@unige.ch

${ }^{\ddagger}$ Associate Professor, LAMPS, UPVD, Perpignan, France, yacoubi@univ-perp.fr

${ }^{\S} \mathrm{PhD}$, Laboratoire de Conception et d'Intégration des Systèmes (LCIS), Grenoble Institute of Technology (Grenoble INP), Grenoble, France, Boussad.Hamroun@lcis.grenobleinp.fr

`Associate Professor, LAGEP, Université Claude Bernard - Lyon 1, Lyon, France, laurent.lefevre@lagep.univ-lyon1.fr

"Professor, LCIS, Grenoble INP, Grenoble, France, Eduardo.Mendes@lcis.grenobleinp.fr 


\begin{abstract}
Numerical simulations of free surface flows are important to provide a prediction tool for the optimal management of irrigation canals. Here we consider an alternative to solving the shallow water equations. We propose a free surface model in which the vertical component of the water current is fully resolved. We believe that such a detailed description can be useful to model the flow around gates or in other situations where the vertical structure of the flow will be important such as in the case of sediment transport and deposition. Our approach is based on a two-fluid Lattice Boltzmann model. We compare the predictions obtained from numerical simulation and experiments performed on a laboratory micro-canal facility.
\end{abstract}

\title{
1 Introduction
}

Many models exist for the simulation of the water flow dynamics in openair channels. They lead to many more simulation tools. A number of the more important ones among these tools have been analyzed and compared in [Clemmens et al., 2005]. Certainly the most popular models for the unsteady flow dynamics in canal reaches are derived from the Navier-Stokes equations using some simplification assumptions. For instance considering an incompressible fluid with constant vertical profiles of the horizontal velocity field leads to the classical Saint-Venant or shallow water equations with free moving surface as considered in many textbooks [Chow, 1985]. 
For simulation or control purposes, such models derived from the NavierStokes equations are further reduced or simplified in some way. A popular simplification neglects the inertia terms in the balance equations and leads to a second order differential equation for the water flows which does not depend on the water level and may be integrated separately (see the diffusive wave model in [Miller and Cunge, 1975]). If transport and diffusion coefficients are assumed to remain constant, this equation reduces to the Hayami model which is linear. The transfer function between upstream and downstream water flows in this Hayami model (which is not a rational fraction since the model is spatially distributed) may still be explicitly computed or approximated by a second order delayed model [Henderson, 1989]. Another simplification relies on the linearization of the Saint-Venant model and on the resulting transfer model which has been proven to be a reasonable approximation of the reach dynamics [Baume et al., 1998]. Finally some control approaches use a "control model" which neglects slope and frictions [Coron et al., 1999], this assumption being reasonable only for some specific applications. Recently models of irrigation systems have also been proposed [Hamroun et al., 2006] which are based on an explicit representation of the power exchanges within the reach and trough the boundaries. They lead to a thermodynamical circuit-like description of the system equivalent to the closed form Shallow Water classical equations derived from the material and momentum balance equations and from the phenomenological constitutive 
equations for friction forces and hydraulic works.

However, although all these models may be efficient for some analysis, simulation and (nonlinear) control purposes, they seem inadequate to handle complex and moving geometries, spatial multi-scale phenomena and coupled multi-phase problems (sediments or algae).

Whatever the chosen continuous model is, either for simulation or control purposes, it has to be reduced to a finite-dimensional model in some way. Among the reduction schemes for the "Saint-Venant-like" models, we may wish to distinguish between total discretization schemes which lead to discrete time models and partial (spatial) discretization schemes leading to continuous time but finite-dimensional approximations. In the first class, the Preissmann implicit finite difference scheme [Cunge et al., 1980] is such a total discretization scheme and has been extensively used both for simulation and control purposes (see [Malaterre and Rodellar, 1997] or [Sawadogo et al., 1995]). Orthogonal collocation is an example of the second class, and has been most frequently used to design a simplified "control" model (see [Dulhoste et al., 2001] for input-output linearization, [Besançon et al., 2001] for backstepping or [Ouarit et al., 2003] for robust optimal control).

However it should be noticed that even in the case of spatial discretization, a time discretization will be finally performed to integrate to system of reduced differential equations.

In this paper we do not propose a continuous time and/or spatial model. 
We rather consider a totally discretized model. The idea is to use the Lattice Boltzmann method [Chopard and Droz, 1998, Wolf-Gladrow, 2000, Succi, 2001] to propose directly a mesoscopic model defined on a discrete time-space lattice. Hence modelling assumptions and model derivation are intrinsically discrete. The starting point for such a model will be again the mass and momentum balance equations which are used in Navier-Stokes or Saint-Venant models. But here these balance equations are "collision rules" written for the particles densities distribution and local momentum. Unlike in classical Lattice Boltzmann model for fluid dynamics (as [Zhou, 2004]) we here consider a bi-fluid air/water model which implements the interaction of two immiscible fluids. Interaction forces between the two kind of fluid particles result in a surface tension and a practical phase separation [Shan and Chen, 1993]. As it will be shown this mesoscopic model appears to be sufficient to recover the desired macroscopic dynamical behavior and naturally takes into account the effect of the air pressure and evaporation. The final objective of such a Lattice Boltzmann model, from our point of view, is to develop a simulation tool able to handle :

- classical long range dynamical effects like water accumulation in reaches, wave propagation resulting in time-varying delay and in some cases wave superposition effects, variable water level profiles due to water bed friction forces, etc.

- algae growth, transport and deposition which are a major problem for 
the operating of some hydraulic works.

- sediment transport phenomena which considerably affect the flow dynamics by modifying the cross section of the reaches.

- local dynamical effects like turbulent dynamics, the existence of both torrential and fluvial water flows around the gates, sediment or algae local accumulation or abrupt removal, etc.

We think that the simultaneous simulation of long range and local range effects (hence slow and fast dynamics) is a major challenge for the unsteady flow modelling in complex irrigation systems. Moreover it allows the definition of new control problems. For instance the work in [Malaterre et al., 1998] classifies the existing control problems which may be addressed using existing classical models. This classification does not consider however the kind of model we develop here, neither the kind of control problems we expect to solve. For instance one may desire to control the reach bottom profile in the presence of sediment transport and deposition or to remove a stack of algae by an appropriate gate action. Investigations on the application of the Lattice Boltzmann method for the simulation of free surface flows are not complete. It will need more work to produce well validated results and practical solutions for complex irrigation system simulation and control problems as those presented for instance in [Clemmens et al., 1998]. We will greatly appreciate receiving comments from hydraulic and irrigation 
engineers with experience and practical problems.

The paper is organized as follows. In the next section the Lattice Boltzmann method is briefly summarized. The mesoscopic mass and momentum balance equations are detailed as well as the water-air interaction forces. The geometry assumptions and boundary conditions are defined for the case of unsteady flows in a single reach. In section 3 the problem of modelling the underflow gates dynamics is addressed. The classical gate equations resulting from the Bernoulli equation are derived for two draining experiments which are defined on a laboratory micro-channel. This experimental setup is briefly presented. Then experimental and simulation results are presented to validate the Lattice Boltzmann approach. Finally concluding remarks summarize the obtained results from the proposed model and sketch some future works and prospects for two-scale local-global modelling and control problems.

\section{Proposed Lattice Boltzmann model}

\subsection{Lattice Boltzmann models}

The Lattice Boltzmann (LB) method is an acknowledged alternative to standard computational fluid dynamics techniques to solve the Navier-Stokes equations and other partial differential equations.

The LB method for hydrodynamics is a mesoscopic approach in which a 
fluid is described in terms of density distributions $f_{i}(\mathbf{r}, t)$ of idealized fluid particles moving and colliding on a regular lattice. These fluid particles can only take a finite number of possible velocities $\mathbf{v}_{i}$ such that, in one time step of the dynamics, particles move from one lattice site to another.

LB models are described in several textbooks or review papers. See for instance [Chen and Doolen, 1998, Succi, 2001, Chopard and Droz, 1998, Wolf-Gladrow, 2000, Sukop and Thorne, 2005].

Among the advantages of the LB method over more traditional numerical schemes, there are its simplicity, its flexibility to describe complex flows and its local nature (no need to solve a Poisson equation). Another benefit of the LB method is its extended range of validity when the Knudsen number is not negligible (e.g. in microflows) [Ansumali et al., 2007].

For regular computational domains, LB solvers can be parallelized very naturally and scale well up to thousands of processors. A few papers analyze the CPU performance, memory usage and accuracy of the LB method in comparison with more classical CFD techniques [Kandhai, 1999, Geller et al., 2006]. Although a general conclusion is difficult to make, it is usually observed that LB model are either comparable to the other methods, or better with respect to the above criteria. In a recent study we show that the LB approach for the $1 \mathrm{D}$ shallow water equation is 100 times faster than an implicit finite difference code, for the same level of accuracy [Pham, 2009, Pham et al., 2010]. Note that the LB method is memory intensive and a 
smart implementation can significantly improve its performance, both in sequential or in parallel (see for instance [Chopard, 2009, Donath et al., 2008, Axner et al., 2008]).

The key quantities of a LB model are the density distributions $f_{i}(\mathbf{r}, t)$ and the "molecular velocities" $\mathbf{v}_{i}$, for $i=0 \ldots z$, where $z$ is called the lattice coordination number of the chosen lattice topology and $z+1$ is the number of discrete velocities. The quantity $f_{i}$ denotes the density of particles entering a lattice site $\mathbf{r}$ at time $t$ with discrete velocity $\mathbf{v}_{i}$. Note that $\mathbf{v}_{i}$ is a vector so that molecular velocities have both a norm and a direction.

For instance, a common choice of velocities in $2 \mathrm{D}$ problems is the socalled D2Q9 topology defined as

$$
\begin{aligned}
& \mathbf{v}_{0}=(0,0) \quad \mathbf{v}_{1}=v(1,0) \quad \mathbf{v}_{2}=v(1,1) \quad \mathbf{v}_{3}=v(0,1) \quad \mathbf{v}_{4}=v(-1,1) \\
& \mathbf{v}_{5}=v(-1,0) \quad \mathbf{v}_{6}=v(-1,-1) \quad \mathbf{v}_{7}=v(0,-1) \quad \mathbf{v}_{8}=v(1,-1)
\end{aligned}
$$

In these expressions, $v$ is a velocity norm defined as $v=\Delta x / \Delta t$, with $\Delta x$ being the lattice spacing and $\Delta t$ the duration of the time step. Both $\Delta x$ and $\Delta t$ can be expressed in any desired unit system.

From the $f_{i}$ 's and the $\mathbf{v}_{i}$ 's we can define the standard physical quantities such as particle density $\rho$, particle current $\rho \mathbf{u}$, and momentum tensor $\Pi_{\alpha \beta}$, 

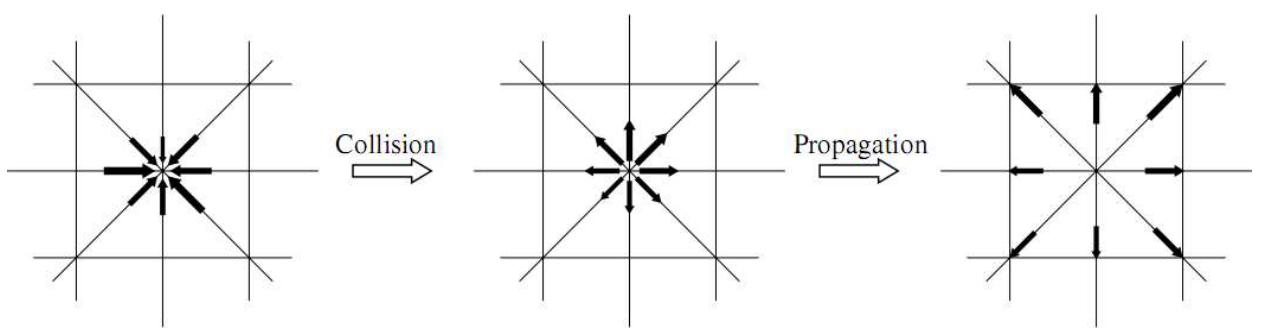

Figure 1: Illustration of the collision and propagation phases for the D2Q9 LB model. The arrows represent the fluid particles, their directions correspond to $\mathbf{v}_{i}$ and their length is proportional to $f_{i}$. Particles at rest (with $\left.\mathbf{v}_{0}=0\right)$ are not shown here.

by taking various moments of the distribution

$\rho(\mathbf{r}, t)=\sum_{i} f_{i}(\mathbf{r}, t) \quad \rho(\mathbf{r}, t) \mathbf{u}(\mathbf{r}, t)=\sum_{i} f_{i}(\mathbf{r}, t) \mathbf{v}_{i} \quad \Pi_{\alpha \beta}=\sum_{i} f_{i}(\mathbf{r}, t) v_{i \alpha} v_{i \beta}$

where Greek subscripts label spatial coordinates.

Following our particle interpretation, we can say that, in a LB model, all particles entering the same site at the same time from different directions (i.e. particles with different molecular velocities $\mathbf{v}_{i}$ ) collide. As a consequence a new distribution of particles results. Then, during the next time step $\Delta t$, the particles emerging from the collision move to a new lattice site, according to their new speed. Therefore, the dynamics of a LB model is the alternation of collision and propagation phases.

This is illustrated in fig. 1, for the D2Q9 lattice topology. In accordance 
with fig. 1, the collision-propagation dynamics can be written as

$$
f_{i}\left(\mathbf{r}+\Delta t \mathbf{v}_{i}, t+\Delta t\right)=f_{i}(\mathbf{r}, t)+\Omega_{i}(f(\mathbf{r}, t))
$$

where $\Omega$ is a model specific function describing the outcome of the particle collision (when subscript $i$ is omitted, $\Omega$ denotes the $(z+1)$-dimensional vector with $\Omega_{i}$ as components).

Although several forms for $\Omega$ exist, the so-called single relaxation time model (also termed Lattice BGK model (LBGK) for its correspondence with the BGK form of the continuous Boltzmann equation [Bhatnager et al., 1954]) is still the most popular collision term. It reads

$$
f_{i}\left(\mathbf{r}+\Delta t \mathbf{v}_{i}, t+\Delta t\right)=f_{i}(\mathbf{r}, t)+\frac{1}{\tau}\left(f_{i}^{e q}-f_{i}\right)
$$

where $f^{e q}$ is called the local equilibrium distribution: it is a given function which depends on the phenomena that we want to model (note that when we refer to all $f_{i}$ or all $f_{i}^{e q}$, we drop the subscript $i$ ). The quantity $\tau$ is the so-called relaxation time. It is a parameter of the model which is actually related to the viscosity of the fluid (see below).

In eq. (4) the local equilibrium distribution $f^{e q}$ depends on space and time only through the conserved quantities. In a hydrodynamic process, where both mass and momentum are conserved, $f^{e q}$ will then be a function of $\rho$ and $\mathbf{u}$.

Thus, in eq. (4), to compute $f_{i}\left(\mathbf{r}+\Delta t \mathbf{v}_{i}, t+\Delta t\right)$ from the $f_{i}(\mathbf{r}, t)$ one first has to compute $\rho=\sum f_{i}$ and $\mathbf{u}=(1 / \rho) \sum f_{i} \mathbf{v}_{i}$ before computing $f_{i}^{e q}(\rho, \mathbf{u})$. 
Afterwards $f_{i}$ can be updated.

It is beyond the scope of this article to show the equivalence between the LB model and the differential equations representing the corresponding physical phenomena. This derivation requires rather heavy mathematical calculations and can be found in several textbooks. See for instance [Chopard and Droz, 1998, Chopard et al., 2002, Lätt, 2007] for a derivation based on the so-called multiscale Chapman-Enskog formalism. Or, see [Junk et al., 2005] for a derivation based on asymptotic expansions. Here we will simply give the important results, without demonstration.

A central ingredient of LB models is to properly enforce the physical conservation laws in the collision term. Hydrodynamics is characterized by mass and momentum conservation which, in the differential equation language, are expressed by the continuity and Navier-Stokes equations.

Conservation laws impose conditions on $f_{i}^{e q}$ when a LBGK model is considered. In order to have $\sum_{i} f_{i}\left(\mathbf{r}+\Delta t \mathbf{v}_{i}, t+\Delta t\right)=\sum_{i} f_{i}(\mathbf{r}, t)$ (mass conservation) and $\sum_{i} \mathbf{v}_{i} f_{i}\left(\mathbf{r}+\Delta t \mathbf{v}_{i}, t+\Delta t\right)=\sum_{i} \mathbf{v}_{i} f_{i}(\mathbf{r}, t)$ (momentum conservation), $f_{i}^{e q}$ must satisfy

$$
\sum_{i} f_{i}^{e q}=\sum_{i} f_{i}=\rho \quad \sum_{i} \mathbf{v}_{i} f_{i}^{e q}=\sum_{i} \mathbf{v}_{i} f_{i}=\rho \mathbf{u}
$$

In addition, in order to recover a hydrodynamic behavior, one imposes that $\Pi_{\alpha \beta}^{e q}$, the second moment of $f^{e q}$, which is the non-dissipative part of the 
momentum tensor, has the standard Euler form

$$
\Pi_{\alpha \beta}^{e q}=\sum_{i} f_{i}^{e q} v_{i \alpha} v_{i \beta}=p \delta_{\alpha \beta}+\rho u_{\alpha} u_{\beta}
$$

where $p$ is the pressure.

Using the properties of the velocity vectors $\mathbf{v}_{i}$, it can be shown (see [Chopard and Droz, 1998, Wolf-Gladrow, 2000, Succi, 2001]) that the following expression for $f^{e q}$ satisfies the conservation laws (5)

$$
f_{i}^{e q}=f_{i}^{e q}(\rho, \mathbf{u})=\rho w_{i}\left(1+\frac{v_{i \alpha} u_{\alpha}}{c_{s}^{2}}+\frac{1}{2 c_{s}^{4}} Q_{i \alpha \beta} u_{\alpha} u_{\beta}\right)
$$

where the $Q_{i}$ 's are tensors whose spatial components are

$$
Q_{i \alpha \beta}=v_{i \alpha} v_{i \beta}-c_{s}^{2} \delta_{\alpha \beta}
$$

and $w_{i}$ are weights which depend on the lattice topology. For a D2Q9 model, and the numbering given in $(1), w_{0}=4 / 9, w_{1,3,5,7}=1 / 9$ and $w_{2,4,6,8}=$ $1 / 36$. Note that in eq. (7) as in what follows, we use Einstein summation convention over repeated Greek indices.

We can also show that the second moment of eq. (7) gives the correct expression for the Euler momentum tensor (6), provided that the pressure is related to the density $\rho$ through an ideal gas relation

$$
p=\rho c_{s}^{2}
$$

where $c_{s}$ is the speed of sound, whose value is related to the lattice topology. For D2Q9, $c_{s}^{2}=1 / 3$. 
From the above result we conclude that the pressure is directly obtained from the numerical scheme, without having to solve a Poisson equation, as it is usually the case when solving Navier-Stokes equations. LB models are slightly compressible models.

Using expression (7) for $f^{e q}$, the behavior of the LB model (4) can be analyzed mathematically with, for instance a Chapman-Enskog method [Chopard and Droz, 1998, Chopard et al., 2002, Lätt, 2007]. Several important results are obtained. It is found that, to order $\Delta t^{2}$ and $\Delta x^{2}$, and for small Mach number $\left(\mathbf{u} \ll c_{s}\right)$, the LB dynamics implies that $\rho$ and $\mathbf{u}$ obey the continuity equation

$$
\partial_{t} \rho+\partial_{\alpha} \rho u_{\alpha}=0
$$

and the Navier-Stokes equation

$$
\partial_{t} \mathbf{u}+(\mathbf{u} \cdot \nabla) \mathbf{u}=-\frac{1}{\rho} \nabla p+\nu \nabla^{2} \mathbf{u}
$$

with a kinematic viscosity $\nu$ depending on the relaxation time $\tau$ as

$$
\nu=c_{s}^{2} \Delta t(\tau-1 / 2)
$$

Finally, in the hydrodynamic regime, it is possible to obtain the expression of $f$ in terms of the hydrodynamic quantities. We write the density distributions $f_{i}$ as

$$
f_{i}=f_{i}^{e q}+f_{i}^{n e q} \quad \text { assuming } f_{i}^{n e q} \ll f_{i}^{e q}
$$

where $f_{i}^{e q}$, by its definition (7) is already a function of $\rho$ and $\mathbf{u}$. 
The Chapman-Enskog expansion then gives (see for instance [Chopard et al., 2002, Lätt, 2007])

$$
f_{i}^{n e q}=-\Delta t \tau \frac{w_{i}}{c_{s}^{2}} \rho Q_{i \alpha \beta} \partial_{\alpha} u_{\beta}=-\Delta t \tau \frac{w_{i}}{c_{s}^{2}} \rho Q_{i \alpha \beta} S_{\alpha \beta}
$$

where $S_{\alpha \beta}=(1 / 2)\left(\partial_{\alpha} u_{\beta}-\partial_{\beta} u_{\alpha}\right)$ is the strain rate tensor. As we can see from this relation, the derivatives of $\mathbf{u}$ are part of the $\mathrm{LB}$ variables.

Note finally that an external force $\mathbf{F}$ (usually called body force) can be added to eq. (4). When $\mathbf{F}$ is constant with respect to space and time variations, the new dynamics reads

$$
f_{i}\left(\mathbf{r}+\Delta t \mathbf{v}_{i}, t+\Delta t\right)=f_{i}(\mathbf{r}, t)+\frac{1}{\tau}\left(f_{i}^{e q}-f_{i}\right)+\frac{\Delta t}{v^{2}} \mathbf{v}_{i} \cdot \mathbf{F}
$$

Note that for non-constant body forces, several ways have been proposed in the literature to modify eq. (4). A popular methods is described in [Guo et al., 2002]. However, in the present work we shall still use (12), knowing that we may thus introduce slight deviation to the Navier-Stokes behavior when $\mathbf{F}=\mathbf{F}(\mathbf{r}, t)$.

\subsection{The bi-fluid open-air canal model}

\subsubsection{The Shan-Chen Model}

In this work, we are interested to simulate the flow of water in a canal. We have chosen to consider a two-fluid LB model to describe the water+air system and to model a free surface flow. Recently, free surface LB models which do not require the explicit simulation of the gas phase have been 
proposed [Ginzburg and Steiner, 2003, Ginzburg, 2005, Koerner et al., 2005, Xing et al., 2007, Ginzburg, 2007, Thürey and Rüde, 2009]. We plan to consider them in a subsequent analysis but here we fully simulate the water and the air above it.

Our two-fluid model is based on the Shan-Chen model for immiscible fluids [Shan and Chen, 1993]. It is a LB model in which each fluid component obeys a LB dynamics, as detailed in the previous section. A coupling is introduced between the two fluids, in terms of a local repulsive force, in order to create an interface between water and air.

In this model, each lattice cell contains two types of populations, denoted $R_{i}(\mathbf{r}, t)$ and $B_{i}(\mathbf{r}, t)$, describing the often called "red" and "blue" fluids, respectively. The density of each fluid is calculated separately from the corresponding distribution functions (see [Martys and Chen, 1996, Shan and Chen, 1993, 1994]):

$$
\begin{aligned}
& \rho_{R}(\mathbf{r}, t)=\sum_{i=0}^{z} R_{i}(\mathbf{r}, t) \\
& \rho_{B}(\mathbf{r}, t)=\sum_{i=0}^{z} B_{i}(\mathbf{r}, t)
\end{aligned}
$$

The calculation of the total momentum at each location, however, has to take into account the momentum of each of the two components [Martys and Chen, 1996] :

$$
u \cdot\left(\frac{\rho_{R}}{\tau_{R}}+\frac{\rho_{B}}{\tau_{B}}\right)=\frac{j_{R}}{\tau_{R}}+\frac{j_{B}}{\tau_{B}}=\frac{1}{\tau_{R}} \cdot \sum_{i=0}^{z} \mathbf{v}_{i} R_{i}(\mathbf{r}, t)+\frac{1}{\tau_{B}} \cdot \sum_{i=0}^{z} \mathbf{v}_{i} B_{i}(\mathbf{r}, t)
$$


where $\tau_{R}$ and $\tau_{B}$ are the relaxation times of species $R$ and $B$, respectively.

An interaction force is introduced between the particles of different kind to mimic their mutual repulsion. The result is the existence of a surface tension between the two fluids. This interaction force is supposed to occur only within the nearest neighbors, and can be expressed as follows :

$$
\begin{aligned}
& F_{R}(\mathbf{r})=-\psi_{R}(\mathbf{r}) G_{R B} \sum_{i=0}^{z} \psi_{B}\left(\mathbf{r}+\Delta t \mathbf{v}_{i}\right) \mathbf{v}_{i} \\
& F_{B}(\mathbf{r})=-\psi_{B}(\mathbf{r}) G_{R B} \sum_{i=0}^{z} \psi_{R}\left(\mathbf{r}+\Delta t \mathbf{v}_{i}\right) \mathbf{v}_{i}
\end{aligned}
$$

Here $\psi_{\sigma}$ for $\sigma=R, B$ denotes the function of the local density $\psi\left(\rho_{\sigma}(\mathbf{r})\right)$ and $G_{R B}<0$ defines the amplitude of the interaction potential. These two expressions are then introduced in the LB dynamics as a body force, as shown in eq. (12).

\subsubsection{The canal model}

Our canal model has been described in previous works (see [Marcou et al., 2006] and [Marcou et al., 2007]). Here we briefly recall the main ingredients.

Our model describes the water flow in an open-air channel. For the sake of simplicity, we consider a vertical cut aligned along the canal axis. Therefore a $2 \mathrm{D}$ free surface model is enough to capture the vertical component of the velocity field.

Starting from a D2Q9 Shan-Chen model, we assume that the red fluid represents the water and the blue fluid the air. In order to make the water 
more heavy than the air and to produce an hydrostatic pressure $p(z)=$ $p_{0}-g z$ in the water, we add a gravity term as an extra body force

$$
\mathbf{F}=(0,-g \rho)
$$

to the LB equation. Note however that this gravity acts only on the water component and ensures that the water remains at the bottom of the pool and the air fluid at the top. As shown in (12) this method produces the expected linear hydrostatic pressure field all over the water layer. Note also that we run our simulation at low Mach number.

The interface between the two phases is usually several lattice sites large. The surface of water is defined at the point at which the densities of the two fluids are equal.

Finally note that our current model does not implement a subgrid model. This can be easily added in a LB model and this addition will be considered in a future development.

\subsubsection{Boundaries}

The second specificity of our model compared to the original Shan-Chen model is the choice of the boundary conditions. We typically want to model one or more adjacent canal sections (reaches), as well as an upstream inlet and a downstream outlet of water. In addition to these inlet and outlet boundaries, we have to define solid walls that prevent flow motion and a socalled "atmosphere" upper boundary for the air component. For illustration, 
a diagram of a lattice for a two-reach system is shown in Fig. 2. Inlet and outlet lattice sites are termed external gates in this figure.

For modelling the walls and the floor, we simply consider the so-called bounce-back boundary condition. It has the main advantage of conserving the mass, even with a hydrostatic pressure gradient at the wall. This is not the case of other, more accurate boundary conditions. See [Marcou et al., 2006, 2007] for a detailed discussion. The bounce-back consists in skipping the ordinary BGK collision and setting the value of each outgoing distribution function at the value of the opposite incoming distribution function.

Atmosphere is modelled by imposing a fixed density to the air at any atmosphere site, and imposing a zero density to the water. Note that if water particles would be present on an atmosphere site, it would then disappear, thus modelling an evaporation process.

Inlet and outlet gates may be modelled with a boundary condition that imposes a desired water discharge $Q_{u p}$ and $Q_{\text {down }}$ at the upstream and downstream boundaries.

In many practical situations, the value of the discharge $Q$ at the inlet and outlet gates is not known a priori. Instead, we know the water heights before the gate (for the upstream inlet), or after the gate (for the downstream one). A constitutive equation relating the drop of water level at the gate and the value of $Q$ must then be used. Such a constitutive equation can be the 


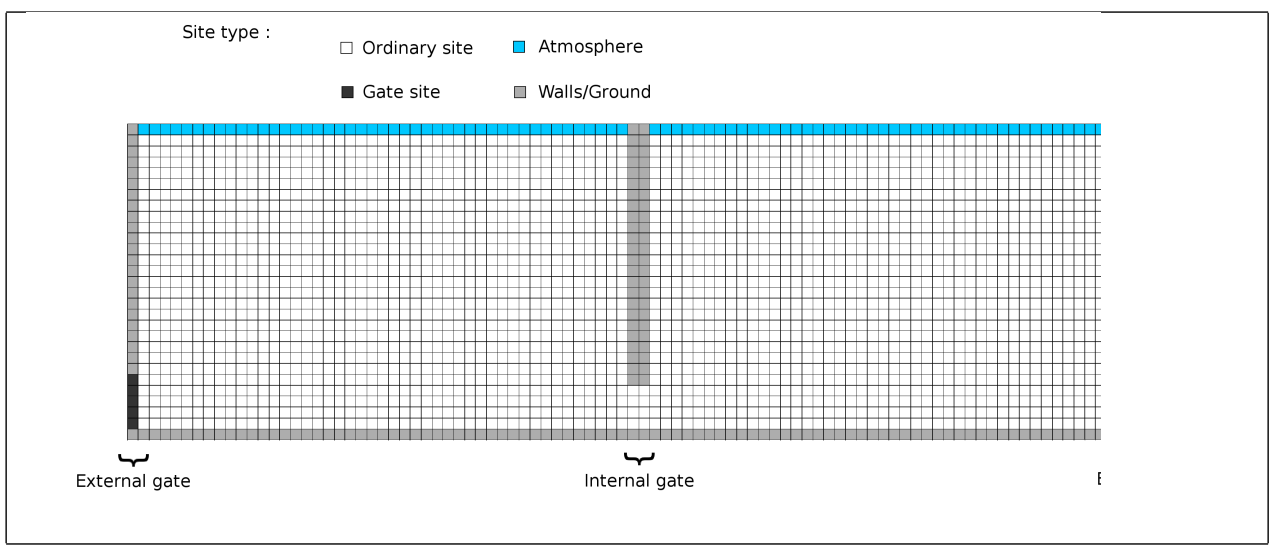

Figure 2: LB computational domain for two open-air canal reaches, with illustration of the different kinds of lattice sites.

well-known gate-equation

$$
Q=\alpha B O \sqrt{2 g} \sqrt{h_{\text {up }}-h_{\text {down }}}
$$

where $\alpha$ is a gate specific coefficient, $O$ its opening and $B$ is the channel width. For an upstream gate, $h_{u p}$ would denote the water level before the gate (given as a boundary condition) and $h_{\text {down }}$ would be the actual water height just after the gate (given by the numerical simulation).

\subsubsection{Parameters choice}

As we saw in the previous sections, the model includes some parameters whose values must be set. These parameters are the time and space steps $\Delta t$ and $\Delta x$, the two fluids relaxation times $\tau_{R}$ and $\tau_{B}$, the gravity $g$, the fluids interaction potential $G_{R B}$ and the form of the interaction function $\psi_{\sigma}$. In the following simulations, we did not seek a quantitative and accu- 
rate correspondence between the simulation results and a well-defined real system, but rather a simple qualitative correspondence between the simulated and experimental results. Indeed, the bi-fluid method we use imposes an artificially large surface tension on the interface between air and water and does not allow us to have the correct density ratio between air and water. These constraints limit the possibilities of calibrating the model so as to make it an accurate simulation of a real water-air free-surface system. Thus, our setting of parameter values is mostly based on numerical stability considerations. Table 1 lists the values of the parameters we used in our simulations.

The choice of the plain Shan-Chen model to simulate open channel flow has been made here mostly for its simplicity and because it implements the phase separation in a physically sound way. It has allowed us to explore the potential of the two-fluid LB approach to provide a new numerical model for irrigation canals. The limitation indicated above needs to be overcome in order to have quantitative predictions that can be applied in real problems. In what follows we have tried to validate our approach by reproducing some behavioral laws rather than detailed features of a given situation. We expect that these behavioral rules (observed when a real canal traverses a gate) reflect the fundamental conservation laws of the systems and, thus, will also be valid when the model parameters are not tuned to their real values. The result shown below in this paper seems to confirm this point of view. 
Other free-surface LB models have been recently proposed in the literature [Ginzburg and Steiner, 2003, Koerner et al., 2005, Xing et al., 2007, Thürey and Rüde, 2009]. The next step is obviously to substitute the current Shan-Chen model with one of them. This is our goal in a forthcoming study. However, it has been noticed by several researchers (including us) that the models of refs. [Ginzburg and Steiner, 2003, Koerner et al., 2005] are not easy to re-implement.

\section{Model Validation}

In the shallow water equations, the vertical scale (i.e. typically the flow depth) is assumed to be much smaller than the horizontal scale. The momentum equation along the vertical direction is then simplified to give the hydrostatic pressure distribution. Although it is still possible to recover approximately the vertical component of the velocity field with this assumption (see [Bates et al., 2005], chapter 10), the most common use of shallow water models neglects the information on the vertical structure of the velocity field. Indeed, near the gates or in the presence of sedimentation phenomena, assuming that the entire column of water moves at the same speed is certainly not satisfying to describe locally the water flow in a canal with some accu-

racy. Another approach exists (see [Stansby and Zhou, 1998]) which incorporates non-hydrostatic pressure distribution in the shallow-water equations and solves the flow field in a 2D vertical plane. 
The free surface LB model we developed also allows a complete description of the vertical component of the velocity field. Thus, it allows us for example to simulate in detail the effect of a gate on the flow. It also allows to model the transport, deposition and erosion of sediments whose deposit clearly has a vertical structure (bumps and lows) which is related to the vertical components of the flow.

Our modelling strategy is thus to use a free surface flow model which takes into account the vertical movement of water when obstacles are present. Such a detailed model can then be coupled with the classical shallow water model in regions where geometry is compatible with the hypothesis of a uniform velocity field in the $z$ direction. Such an approach is typical of multi-scale modelling, for which some parts of the system have to be described with more detailed numerical models.

In this paper, we focus on the flow behavior near a submerged gate in order to validate our free surface LB model. We expect the gate to influence the discharge in function of its geometry and opening.

Since we do not have analytical results on the velocity field around the gate, our validation is based on a comparison between the numerical simulations of the LB model and experiments realized with the micro-canal of the Laboratoire de Conception et d'Intégration des Systèmes (LCIS, Grenoble Institute of Technology). The latter allows us to realize water levels and discharge measurements in reaches separated by gates whose opening is 
adjustable either manually or according to an automatic control system.

In our experiments, we mainly consider the relationship which exists between the water levels before and after the gate $\left(h_{u p}\right.$ and $\left.h_{\text {down }}\right)$ and $Q$, the resulting discharge. Our observations, both on the micro-canal and on numerical simulations, indicate that this relationship depends on the flow regime.

In a regime where a stationary flow is established, the observed behavior is that of eq.(19).

On the other hand, if we consider a transient regime where there is no permanent flow, the observed relationship changes. Practically, when we run a draining experiment (considering two pools connected to each other so that one of them is emptying into the other one), we observe that

$$
Q=\beta O\left(h_{u p}-h_{\text {down }}\right)
$$

As far as we know, this linear relationship is not acknowledged by the open channel community, although it has to be noticed that non parabolic behavior may de derived from eq.(19) if the gate parameter is varying with the gate opening $O$ and the head differential $h_{u p}-h_{\text {down }}$. This dependance has been proved and investigated in [Lozano et al., 2009] for a similar geometry of the sluice gate but different flow regimes.

In this paper we describe experimental and simulation results in these two regimes. Our experimental data show that these two regimes are actually well differentiated and that the numerical model captures them cor- 
rectly.

\subsection{Presentation of the Experimental Micro-Channel}

Experimental results which are presented in this paper and compared to the LB simulation data were obtained with the experimental micro-channel located at the LCIS laboratory in Valence, France (http://lcis.grenobleinp.fr/).

The micro channel is a completely instrumented platform used to reproduce the flows in irrigation canals on a reduced scale. It is illustrated in Fig. 3. The channel length is seven meters and it is supported by a metal lattice girder. The channel part is connected to an upstream tank which acts as source and whose level is maintained constant, a downstream tank and an intermediate tank which is used as tank of storage. The slope of the channel can be modified thanks to a mechanical jack as shown in Fig. 3. The channel has three gates; upstream, downstream and a third in the center makes it possible to experiment the case of interconnected reaches. These gates are actuated by DC motors. They are rectangular sluice gates with sharp edges of about 0.5 centimeter thick. They may be considered rigid and sliding perpendicularly to the flow. All the reach width is left free for the flow. It may be noticed that the geometry of the gates is similar to the ones studied in [Lozano et al., 2009] where a complete study of the conventional discharge equation is performed. In this work the dependance of 
the discharge coefficient with the gate opening and the head differential are proved. This dependance may lead to apparently non parabolic relations between the discharge and the head differential depending on the operating conditions. This is indeed the case for the experiments presented in the sequel. Similar considerations apply for the final downstream weir (outfall) which is also realized with a 0.5 centimeter thick piece of plexiglass with sharp edge fixed perpendicularly to the flow.

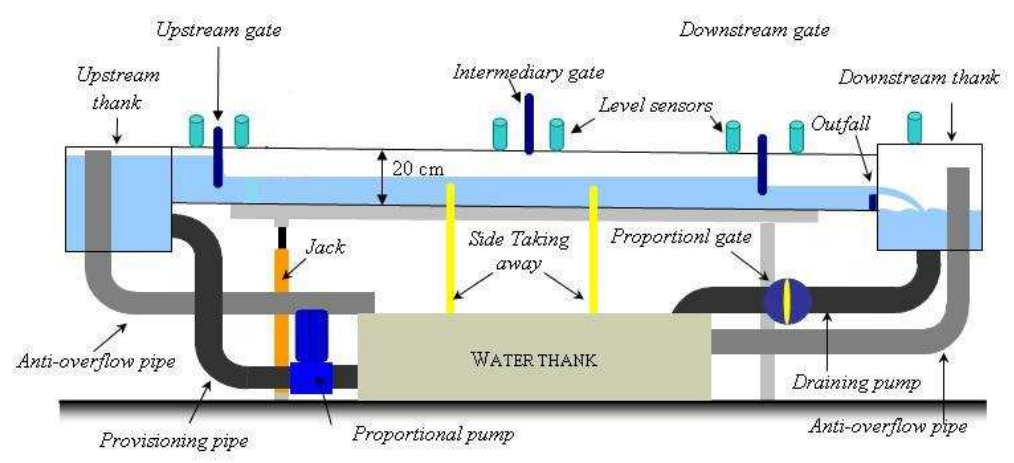

Figure 3: Experimental Micro-Channel

Ultrasonic sensors return a voltage ranging 0-10 Volts which gives an image of the height of water levels in the places where they are placed.

A proportional valve and a pump are used respectively to ensure a constant water level at downstream and upstream tanks of canal. They are controlled by Crouzet automata of the type Millenium II. By mean of a 


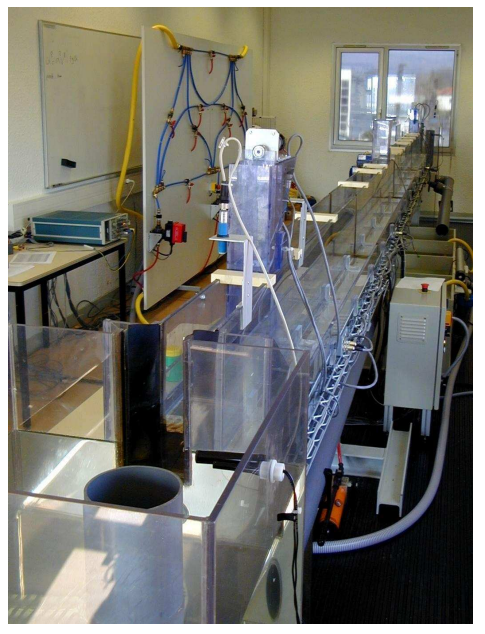

Figure 4: Sight of the experimental Micro-Channel

dSPACE electronic card, all the data from the sensors are recorded on a computer in order to be analyzed. In turn the computer, using the same device, can control the actuators of the micro channel. The parameters of the micro-channel are given in Table 2). A picture of the experimental plant may be viewed on (Fig.4)

\subsection{Draining experiment}

\subsubsection{Description and theory}

The purpose of this experiment is to study the discharge $Q$ at the gate in the situation depicted in Fig. 5. The left pool is initially filled with a water level $h_{\text {init }}$ larger than in the right pool, whose water level $h_{\text {down }}$ is kept almost constant thanks to the outfall. When the gate separating the two pools is open at time $t=0$, the water flow from the left container to the 


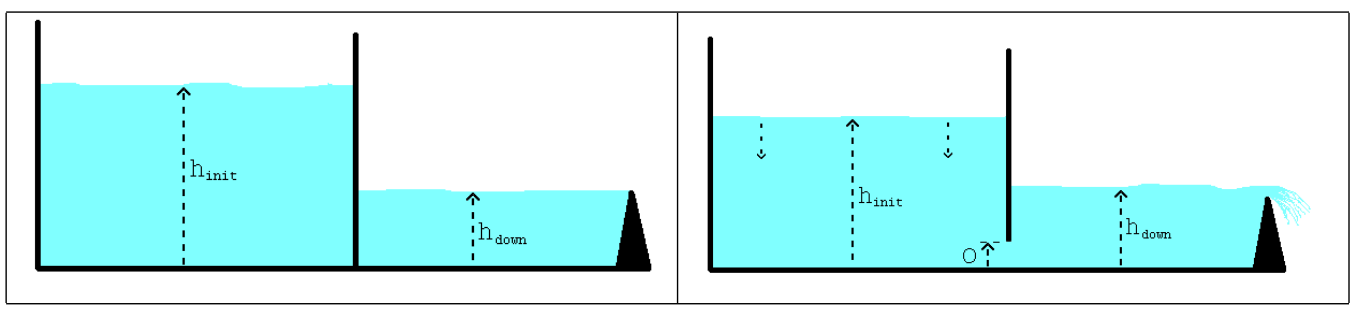

Figure 5: Draining experiment - Diagram of the experiment. Two pools are connected with each other. At the beginning of the experiment, the gate between them is opened to produce a $6 \mathrm{~mm}$ clearance. In the downstream pool, the presence of an outfall $(6 \mathrm{~cm}$ high) ensures that the variations of the downstream pool water level are small.

right one. In both the experiment and the numerical simulation, we measure the water level $h(t)$ in the upstream pool. We assume that the water level is uniform in each pool: $h(x, t)=h(t)$. This approximation is acceptable if the flow is small enough and the length of the pool short enough. We have checked that this assumption is valid on the micro-canal with a pool of length $1.75 \mathrm{~m}$ and the typical magnitude of the discharge $Q$ we used. On the numerical simulations, we have also checked that the water level is essentially horizontal within our parameters range.

Clearly, the behavior of $h(t)$, as a function of time, depends on the discharge $Q(t)$ at the gate. We can compute an analytical expression for $h(t)$ under the assumption that either equations (19) or (20) holds. By comparing these analytical expressions of $h(t)$ with experimental data, we will be able to decide which gate equation is correct for this particular experiment. 
Along with the hypothesis of negligible variations for the downstream water level, the standard gate equation (19) yields the following analytical expression for the evolution of the water level :

$$
\begin{array}{r}
Q=-B L \dot{h}(t) \quad \text { (mass conservation in the pool) } \\
Q=B \alpha O \sqrt{2 g\left(h(t)-h_{\text {down }}\right)} \quad \text { (classical gate equation) }
\end{array}
$$

where $L$ is the length of the pool. The dynamics of the water level is then described by

$$
\dot{h}(t)=a \sqrt{h(t)-h_{\text {down }}}, \quad a=\frac{-\alpha \cdot O \sqrt{2 g}}{L}
$$

For $0 \leq t \leq 2 b / a$, the solution is

$$
h(t)=h_{\text {down }}+\left(-\frac{a}{2} \cdot t+b\right)^{2}
$$

Thus, the analytic solution for the water level in this experiment is a parabola. It is obvious that this equation is only valid until an instant $t_{\max }=2 b / a$ where both water levels become equal, after what $h(t)$ stops its decrease and remains constant (in real systems, however, $h_{\text {down }}$ is not absolutely constant, which rules out such an unphysical termination condition).

We can now perform the same calculation using gate equation (20). We obtain the following equation for $h(t)$

$$
\dot{h}(t)=-\gamma\left(h(t)-h_{\text {down }}\right), \quad \gamma=\beta O L>0
$$

and the solution is (valid for all times $t$ )

$$
h(t)=h_{\text {down }}+\left(h(0)-h_{\text {down }}\right) e^{-\beta t}
$$


Therefore with the linear gate equation, $h(t)$ has an exponential behavior instead of a parabolic one.

\subsubsection{Experimental observations}

Fig. 6 shows the results of one experiment, done on the micro-canal. This result is representative of several of the experiments we conducted. On the left panel, we clearly see the fast decrease of the water height in the left pool (blue curve) and we also observed the slight increase of the level in the right pool, which shows that our approximation which consists in considering $h_{\text {down }}$ constant, may not be good enough. We will come back to this problem at the end of this section.

On the right panel of Fig. 6, the relationship between $\dot{h}(t)$ and the difference of water levels around the gate $\Delta h$ is shown. The quantity $\dot{h}(t)$ is computed by a numerical differentiation of $h(t)$, after smoothing but it is

still very noisy. Due to this noise, which is difficult to filter out (some slightly better results could be obtained with the method described in [Chartrand, 2005]), it is hard to decide between the linear or quadratic gate relation, based on this graph.

As a better check, less sensitive to noise, we compare in Fig. 7 the smoothed value of $h(t)$ with the parabolic fit (right) and the exponential fit (left). Although visually very similar, we can see from the error of the fit that the exponential function corresponds better to the experimental data 

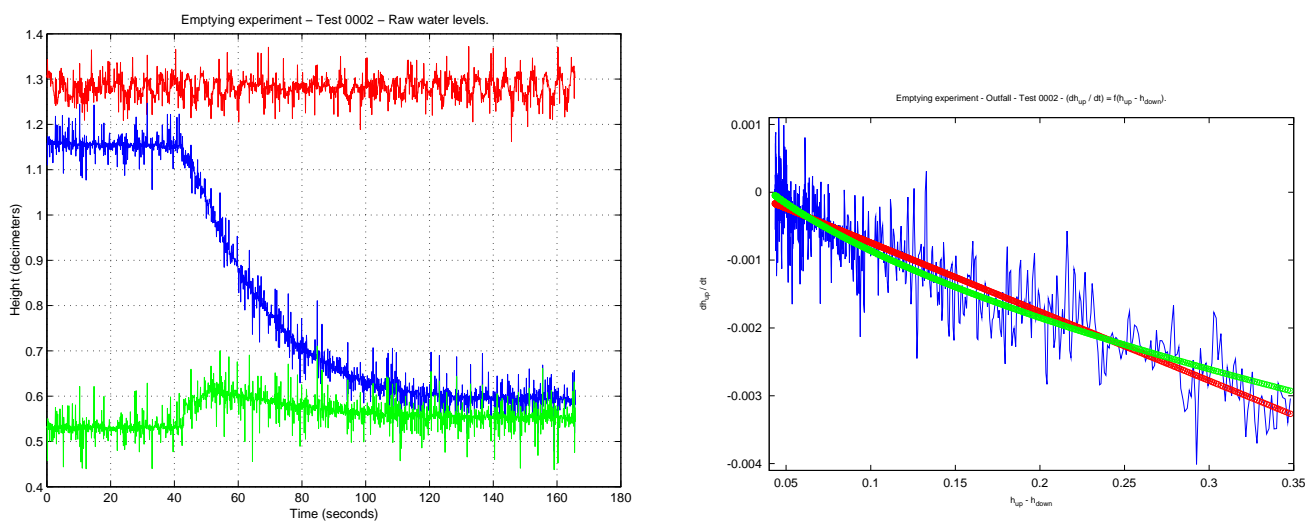

Figure 6: Draining experiment - Left : raw water levels. The blue curve is the water level in the upstream pool. The green curve is the water level in the downstream pool. The red curve is the water level in a reservoir located upstream of the upstream pool, which is used to initialize the water level in the upstream pool before the experiment. Right : dependency between $\dot{h}(t)$ and $\Delta h$. Here $\Delta h$ is computed from the actual values of $h_{u p}$ and $h_{\text {down }}$. Since $\dot{h}(t)$ is proportional to the discharge, this curve shows the relationship between the discharge and the difference of water levels. We have superimposed to the numerical derivative of $h(t)$ the fits obtained assuming either the linear gate equation (straight line) or the standard gate equation (curved line). Due to the numerical noise on $\dot{h}(t)$, it is hard to discriminate the two behaviors. 
than the parabolic function. The same observation is found when repeating the experiment several times. This suggests that the classical gate equation does not apply perfectly in this case.

Another test to check whether the classical gate equation is obeyed or not is to compare the value of gate coefficient $\alpha$ obtained from the parabolic fit with its reference value $\alpha_{r e f}$ known for the micro-canal. From (23) we have

$$
\alpha=-\frac{a L}{O \sqrt{2 g}}
$$

We calculated $\alpha$ for several draining experiments, including those where the parabolic fit seemed better. We found values of $\alpha$ which range between 0.2 and 0.3. In previous works done on the micro-canal [Chaussinand, 2003], the gate coefficient $\alpha_{\text {ref }}$ of the micro-canal was generally found to have values between 0.6 and 0.7 . This reference values have been obtained from stationary flow experiments for which we know that the gate operates accordingly to the classical gate equation and by varying the gate opening in the 1-7 $\mathrm{cm}$ range, close to the $0.6 \mathrm{~cm}$ opening used in the draining experiment.

It suggests that even if we assume that the gate obeys the classical equation, the coefficient $\alpha$ we measure is not right. Note that according to [Lozano et al., 2009], we may expect a variation of $\alpha$ as the gate opening changes. However, the data reported in [Lozano et al., 2009] and the steadystate calibration of our micro-canal tends to exclude the large variation of $\alpha$ we measure here. 


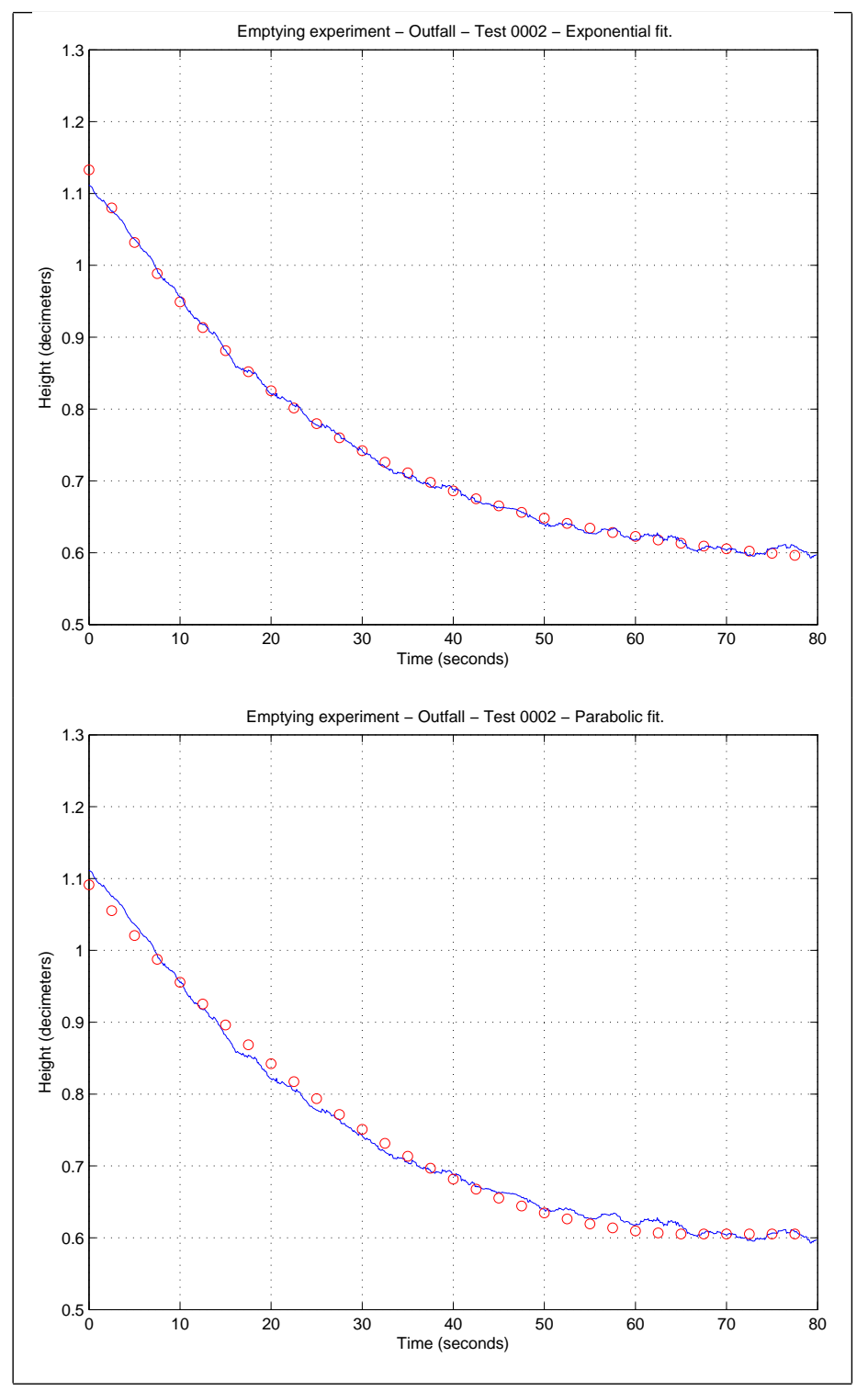

Figure 7: Draining experiment - Left : exponential fit of the experimental water level in the upstream pool. Right : parabolic fit. For both figures, the blue, continuous lines represent the experimental data, while the red circles represent the fit. Relative error with parabolic fit : 0.0147 ; relative error with exponential fit : 0.0059. Gate coefficient calculated from the parabolic fit coefficient : $\alpha_{\text {num }}=0.3057$ 


\subsubsection{Numerical simulations}

Similar tests were done using LB simulations. The simulated system consists in three pools. The first pool (the upstream pool) is the pool whose water is drained during the simulation. The water of this first pool flows to the second one (the downstream pool) through an internal gate.

In turn, the downstream pool communicates with a third pool (the evacuation pool) through an outfall which evacuates excess water from the downstream pool. This third pool contains a number of lattice sites with a specific boundary condition that absorb the water that reach them. The water levels in the upstream and downstream pools are recorded during the simulation, as well as the discharge $Q$ at the gate.

Results for these simulations are shown in Fig. 8 (water level evolution and relation between $\dot{h}(t)$ and $\Delta h=h(t)-h_{\text {down }}$ ) and Fig. 9 (exponential and parabolic fits). The case shown in these figures exhibit an exponential behavior for the evolution of the water level. We can see that there are small variations of the downstream water level, but not very important. The discharge is approximately linear according to the water level difference. The exponential fit is clearly better than the parabolic one. The simulation shown on these figures was done with a $1000 \times 100$ lattice. Note that we have also considered simulations on a $2000 \times 200$ lattice. The results obtained confirm that the exponential fit is better than the parabolic one: on the larger lattice, the error on the exponential fit decreases by 4 compared to 
the smaller one, whereas the error on the parabolic fit slightly increases.

Thus the conclusions from the LB simulations are approximately the same as those from the experiments on the micro canal: during the draining, the classical gate equation does not seem to apply.

\subsubsection{Non-constant $h_{\text {down }}$}

Now, as suggested before, we will reconsider our assumption that the water level $h_{\text {down }}$ is constant during the draining experiment. Fig. 6 clearly shows that the downstream pool level actually varies strongly at the beginning of the experiment and less afterwards.

Our neglecting of this variation of $h_{\text {down }}(t)$ could explain why we did do not obtain the correct $\alpha$ value from that parabolic fit.

Below we show the calculations that take into account these variations $\Delta h_{d}$ of the water level in the downstream reach (still assuming that this water level is uniform in the reach). These calculations are based on the mass balance in the two reaches and on the classical gate equations for, respectively, the underflow gate between the two reaches and the outfall of the downstream reach. Thus we have $h_{\text {down }}=h_{\text {outfall }}+\Delta h_{d}$, where $h_{\text {outfall }}$ is the height of the weir. The discharge $Q_{w}$ over the outfall is assumed to be given by the standard constitutive equation [Graf, 1993]

$$
Q_{w}=\beta B \sqrt{2 g}\left(\Delta h_{d}\right)^{3 / 2}
$$



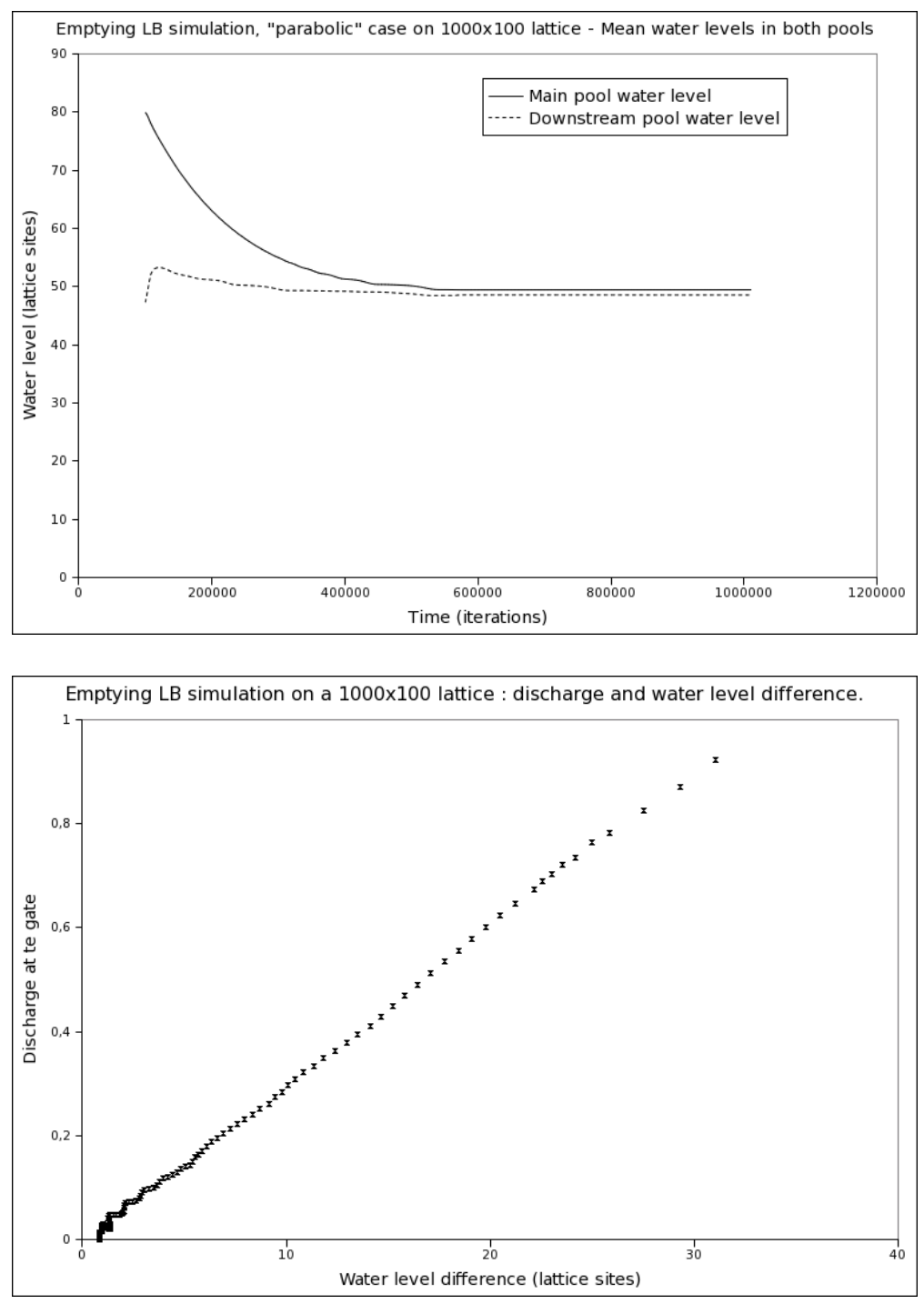

Figure 8: Draining experiment - LB Simulation on a $1000 \times 100$ lattice. Up : evolution of the water level for both the upstream and the downstream pools. Down : dependency between discharge and $\Delta h$. As in the experimental case, the dependency is linear. 


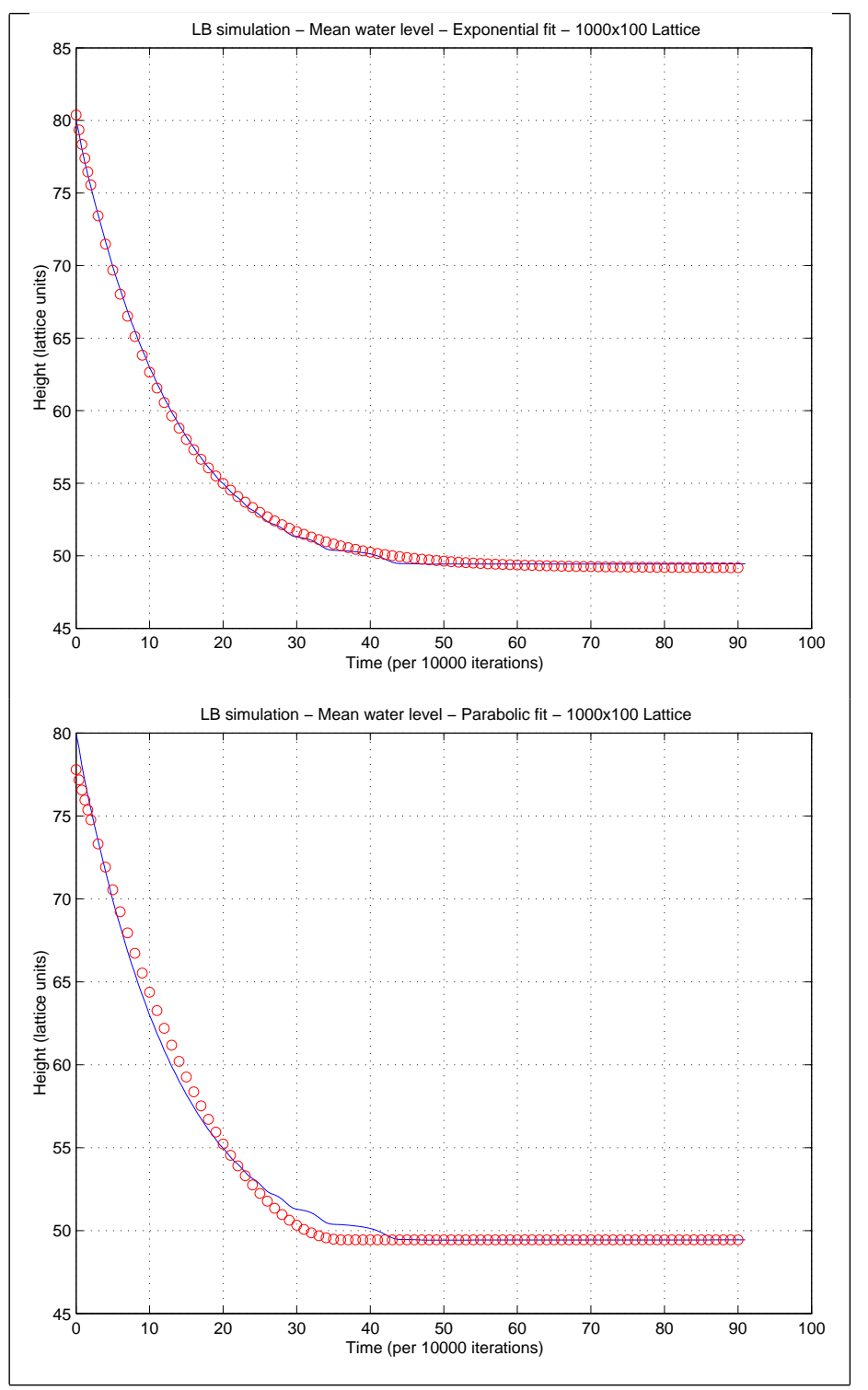

Figure 9: Draining experiment - LB Simulation on a $1000 \times 100$ lattice. Left : exponential fit of the simulated water level in the upstream pool. Right : parabolic fit. The blue, continuous lines represent the simulation data, while the red circles represent the fit. Error with parabolic fit : 0.0071; Error with exponential fit : 0.0041 . The exponential fits the simulation data better. Simulations performed on a $2000 \times 200$ lattice give an error of 0.001 for the exponential fit and 0.0086 for the paßæbolic fit. This provides even stronger support for the validity of the linear gate equation over the quadratic equation. 
Therefore, the discharge $Q_{g}$ at the underflow gate is now

$$
Q_{g}=\alpha B O \sqrt{2 g} \sqrt{h_{u p}-\left(h_{\text {outfall }}+\Delta h_{d}\right)}
$$

and the mass balance equations read

$$
\begin{array}{r}
B L_{1} \dot{h}_{u p}=-Q_{g} \\
B L_{2}\left(\Delta \dot{h}_{d}\right)=Q_{g}-Q_{w}
\end{array}
$$

The quantities $h_{u p}, L_{1}$ are the water level and the length of the main reach and $L_{2}$ is the length of the downstream reach. $h_{u p}$ and $h_{\text {down }}$ both vary during the experiment while $h_{\text {outfall }}$ is a constant.

After some algebra, we obtain the following equations :

$$
\begin{array}{r}
\dot{h}_{u p}=-O \frac{\alpha \sqrt{2 g}}{L_{1}} \sqrt{h_{u p}-\left(h_{\text {outfall }}+\Delta h_{d}\right)} \\
\left(\Delta \dot{h}_{d}\right)=\frac{\alpha \sqrt{2 g}}{L_{2}}\left(O \alpha \sqrt{h_{u p}-\left(h_{\text {outfall }}+\Delta h_{d}\right)}-\beta\left(\Delta h_{d}\right)^{3 / 2}\right)
\end{array}
$$

These equations do not provide a direct analytical expression for the water levels. However, we can obtain the corresponding theoretical evolution for the water levels numerically, after obtaining the $\alpha$ and $\beta$ coefficients via the Newton-Raphson optimization method.

After applying this new fitting method to the micro-canal experiment whose results were showed in Figs. 6 and 7, we obtain the values 0.2731 and 0.3276 for respectively $\alpha$ and $\beta$. Thus, the value for $\alpha$ is very similar to the one we found with the less accurate parabolic fit. An example of the fit realized using these more accurate equations is shown in Fig 10. 


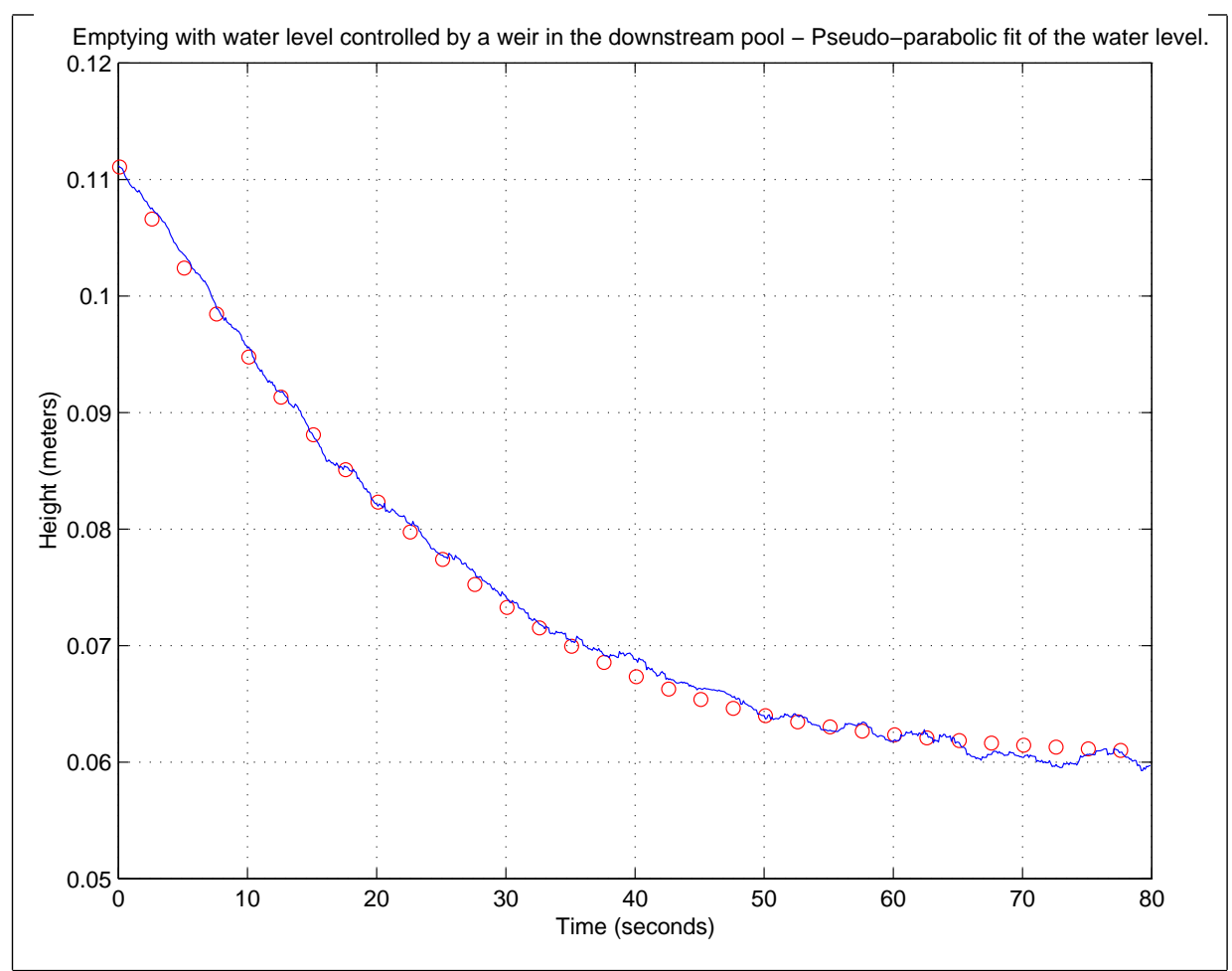

Figure 10: Draining experiment - Fit realized on the same experimental data as those shown on Fig. 6 and 7 by using equations 32 and 33. The values of the coefficients $\alpha$ and $\beta$ are 0.2731 and 0.3276 . The average relative error is 0.0142 , which is very close to the value 0.0147 of the relative error for the ordinary parabolic fit. 
Note that we could have repeated the same calculation also with the linear gate equation. However, since the above result shows that the variation $\Delta h_{d}$ of the water level in the downstream pool has a negligible effect on the fit, we do not expect that the quality of the exponential fit will change in a relevant way.

We can thus conclude that during the draining experiment, the linear gate equation applies better than the quadratic one, whether or not we take into account the variation of $h_{\text {down }}$. The LB model we proposed recovers this observed experimental behavior. As it will be shown in the next section, this LB model also recovers the classical gate equation when the usual stationary flow conditions are considered.

\subsection{Validation of the classical gate equation in a stationary experiment.}

Let us consider the situation described in Fig. 11. A main pool is separated from an upstream and a downstream reach by two gates, whose openings are $O_{u p}$ and $O_{\text {down }}$, respectively. We assume that the water level $h_{\text {pool }}$ in the central pool is uniform and that the $\alpha$ coefficients of both gates are the same. Then, using the classical gate equation (19), the expressions for the discharge $Q_{u p}$ at the left gate and $Q_{\text {down }}$ at right one are as follows :

$$
\begin{array}{r}
Q_{\text {up }}=\alpha O_{\text {up }} \sqrt{2 g} \sqrt{h_{\text {up }}-h_{\text {pool }}} \\
Q_{\text {down }}=\alpha O_{\text {down }} \sqrt{2 g} \sqrt{h_{\text {pool }}-h_{\text {down }}}
\end{array}
$$




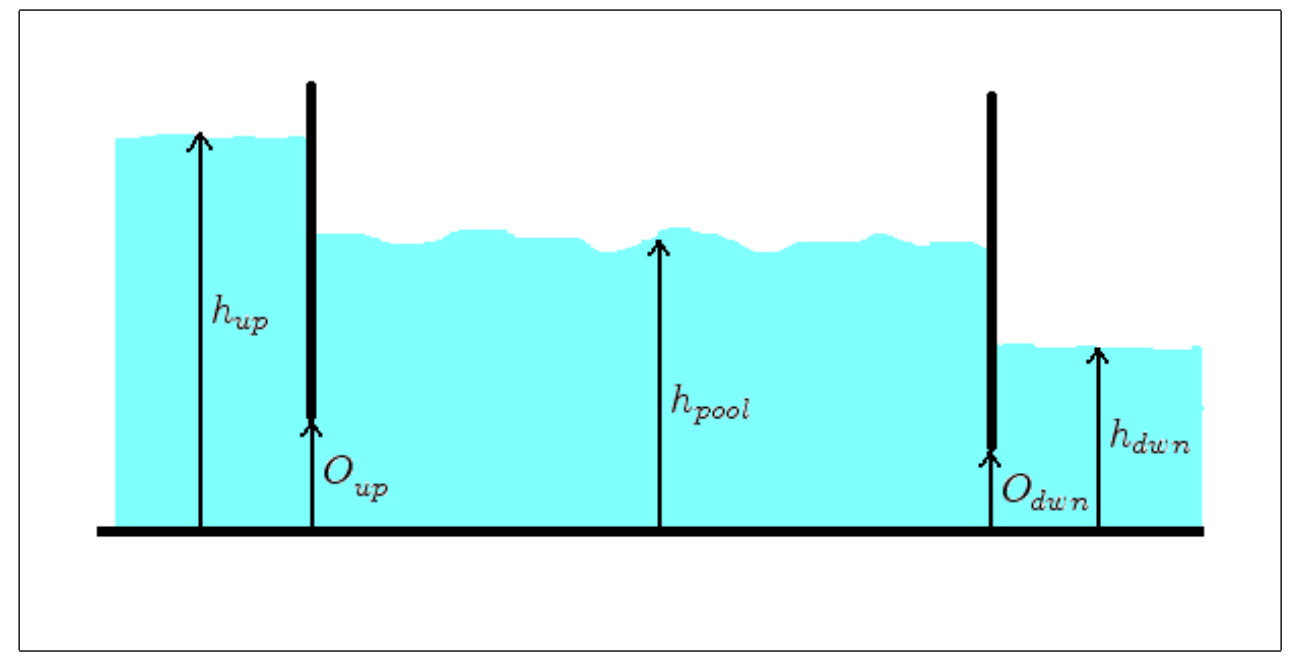

Figure 11: Diagram of the stationary experiment. We consider the equilibrium water level in a reach, as a function of the water levels of the two connected reaches and of the gate openings.

where $h_{u p}$ and $h_{\text {down }}$ denote the water level in the leftmost and rightmost pools.

If $h_{u p}$ and $h_{\text {down }}$ are maintained constant, the system eventually reaches a steady state (that we call here "equilibrium"), in which the discharge at both upstream and downstream gates are equal. This equilibrium condition $Q_{u p}=Q_{\text {down }}$ leads to the following equation :

$$
O_{u p} \sqrt{h_{u p}-h_{\text {pool }}}=O_{\text {down }} \sqrt{h_{\text {pool }}-h_{\text {down }}}
$$

Thus, we obtain the following value for the equilibrium water level $h_{\text {pool }}$ in central reach, as a function of the gates openings and water levels in the 
upstream and downstream reaches :

$$
Q \propto O \sqrt{\Delta h} \Longrightarrow h_{\text {pool }}=\frac{O_{u p}^{2} h_{u p}+O_{d o w n}^{2} h_{\text {down }}}{O_{u p}^{2}+O_{\text {down }}^{2}}
$$

We can also derive a similar relation assuming that the discharge at the gate obeys the linear relation (20). From

$$
Q_{u p}=\beta O_{\text {up }}\left(h_{\text {up }}-h_{\text {pool }}\right)=Q_{\text {down }}=\beta O\left(h_{\text {pool }}-h_{\text {down }}\right)
$$

we conclude that the equilibrium value of $h_{\text {pool }}$ should obey (if the linear relation applies)

$$
Q \propto O \Delta h \Longrightarrow h_{\text {pool }}=\frac{O_{u p} h_{u p}+O_{\text {down }} h_{\text {down }}}{O_{u p}+O_{\text {down }}}
$$

We will now verify from the micro-canal experiments which one of the formula (37) or (39) best fits the observed water level $h_{\text {pool }}$. We note that for both of these formula, the gate coefficient do not play a role as long as both gates are identical.

The experimental setting reproduces the situation of Fig. 11. Note that

- the water level $h_{u p}$ in the upstream reach is externally controlled to remain constant

- the water level $h_{\text {down }}$ in the rightmost reach is fixed due to an outfall

- the equilibrium water level $h_{\text {pool }}$ in the main reach is recorded

- the gate openings $O_{u p}$ and $O_{\text {down }}$ can be adjusted to different values, thus producing different values of the equilibrium water level $h_{\text {pool }}$. 


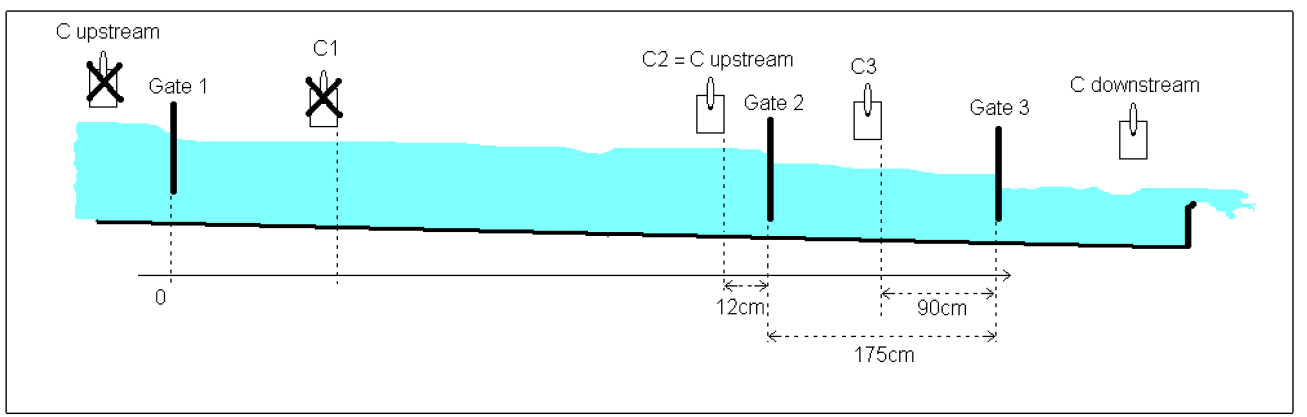

Figure 12: Diagram of the stationary experiment on the micro-canal. The small-sized studied reach in this experiment is the one between gates 2 and 3. Only three of the five available sensors are used during this experiment. A first series of experiments was done on the entire micro-canal, with sensors recording the equilibrium water level at several points of the main reach. However, we observed that the hypothesis of uniformity of the water level which must be verified for our experiment was not satisfied in this case. Thus, further tests were done on a smaller reach where the water level can be considered almost uniform (see Fig 12).

Fig. 13 shows the results obtained for one of these stationary experiments. In this particular set of tests, we fixed $O_{\text {down }}$, while varying $O_{u p}$, the opening of the upstream gate. In this figure, the experimental data of a sensor giving the water level at the middle of the reach as a function of the upstream gate opening are shown $(+$ marks $)$. They are compared with the theoretical values for $h$ computed from equations (37) and (39). The dashed line assumes the classical gate equation and the dotted line assumes the gate linear equation. 
We observe that the best fit of the experimental data is clearly obtained for the classical gate equation (square root dependency between the discharge and the water level difference). It means that the classical gate equation is obeyed when the canal operates in a stationary regime.

In order to reproduce these micro-canal experiment with LB simulations we had to consider a system with three reaches and four gates. The two gates at the far ends of the computational domain are inlet and outlet gates. They impose given flow conditions, as explained in section 2.2.3: we assume fixed water heights in the non-simulated canal reaches, just before the inlet gate and just after the outlet gate, respectively. This procedure allows us to mimic the natural functionning of reals channels and the experimental setting.

If, for instance, a constant discharge was used instead, it could cause some numerical problems. Indeed, in some situations, the water level in the upstream simulated reach could increase beyond the top of the lattice (for example if the upstream internal gate has a very small opening or is closed) before the rest of the system adjusts itself so that the imposed discharge is realized.

On the other hand, the two "internal" gates are simply modelled as holes placed at the bottom of the vertical wall separating the pools. The height of the hole defines the opening of the gate. No additional modelling feature is required. Note that we proceed similarly to build the gate for the draining 
experiment, in the previous section.

The water level in the reach located between the two internal gates is studied. We observe the variations of the equilibrium water level according to the changes in the parameters values (that is, in our case, the changes of the opening of the internal gate located at the upstream end of the observed reach).

In all our simulations, the same parameter values were used for the water levels outside the system and for the gate openings, except for the upstream internal gate, whose value was different for each simulation. This allowed us to obtain the relation between the equilibrium water level and the upstream gate opening. The plot is shown in Fig. 14.

We can see that the theoretical curve corresponding to the classical gate equation seems to fit better the simulation data than the curve based on the linear gate equation, as in the case of the micro-canal experiment.

The conclusion of this section and the previous one is that the gate equation depends on the flow regime and that the LB simulations reproduce this subtle effect. To better understand the distinction between the two regimes (steady flow through the gates or draining experiment), the flow pattern can be compared in the numerical approach. Figs. 15 and 16 show a snapshot of the velocity field as encountered in LB simulations for the two cases. We clearly observe a qualitative difference between the flow patterns. In the steady state case, we observe swirls that are absent in the draining process. 


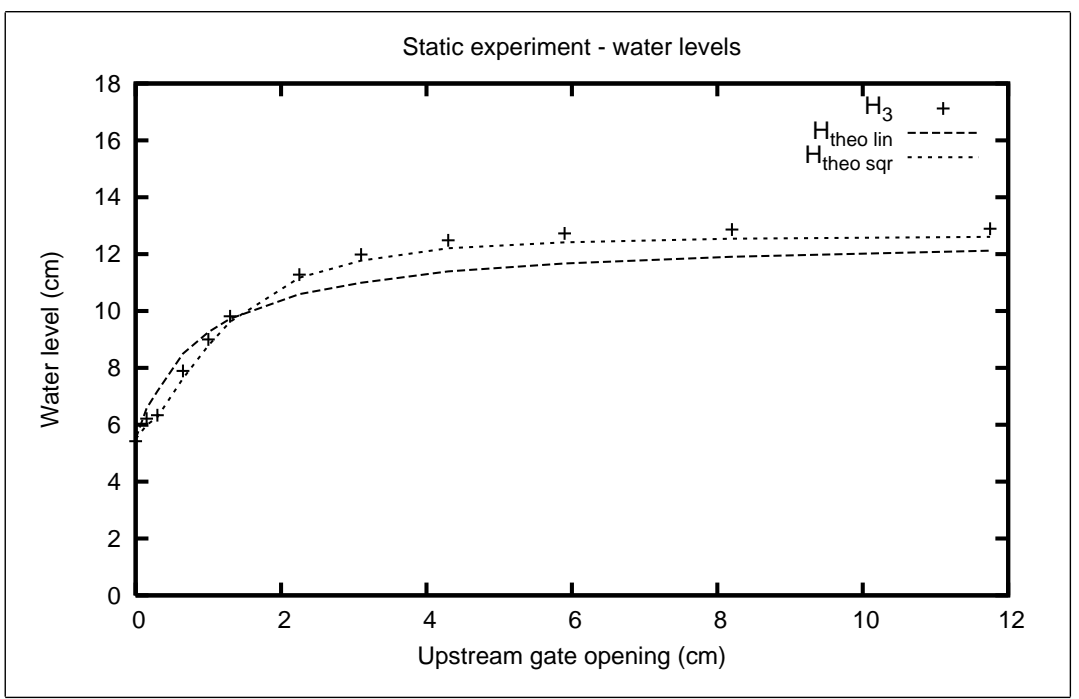

Figure 13: Verification of the theoretical formulas determining the equilibrium water level, in the case of a short reach for the experimental microchannel. The equilibrium water level was measured for several experiments. For each experiment, the opening of the upstream gate was changed. The water levels in each of the three reaches was then recorded. We compare in this figure the recorded experimental equilibrium water level in the studied reach and its theoretical value, calculated according to the gate equations (37) and (39) using measurements for the gate openings and water levels for the upstream and downstream reaches. 


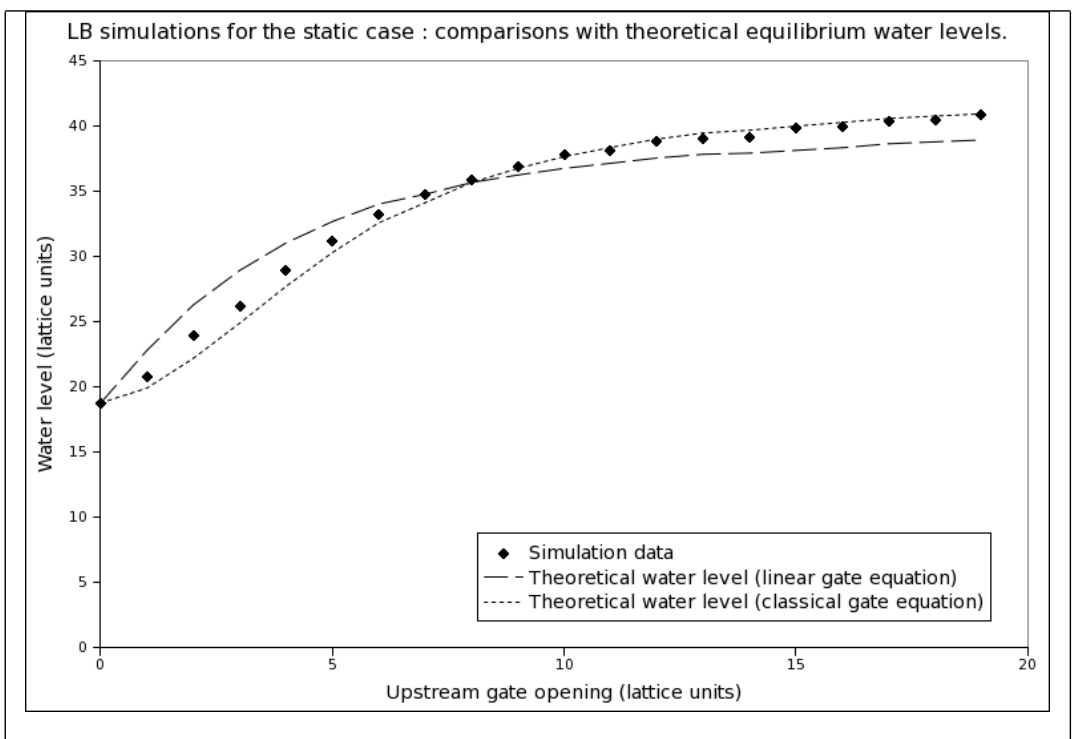

Figure 14: Verification, with the LB model simulations, of the theoretical formulas determining the equilibrium water level in stationary regime with two open gates for the same conditions as used to collect experimental data. The figure shows the simulated equilibrium water level in the studied reach for several opening of the inner upstream gate. The theoretical curve corresponding to the classical gate equation seems to fit better to the simulation data. The upstream and downstream water levels in the non-simulated reaches are respectively of 50 and 20 lattices sites. 


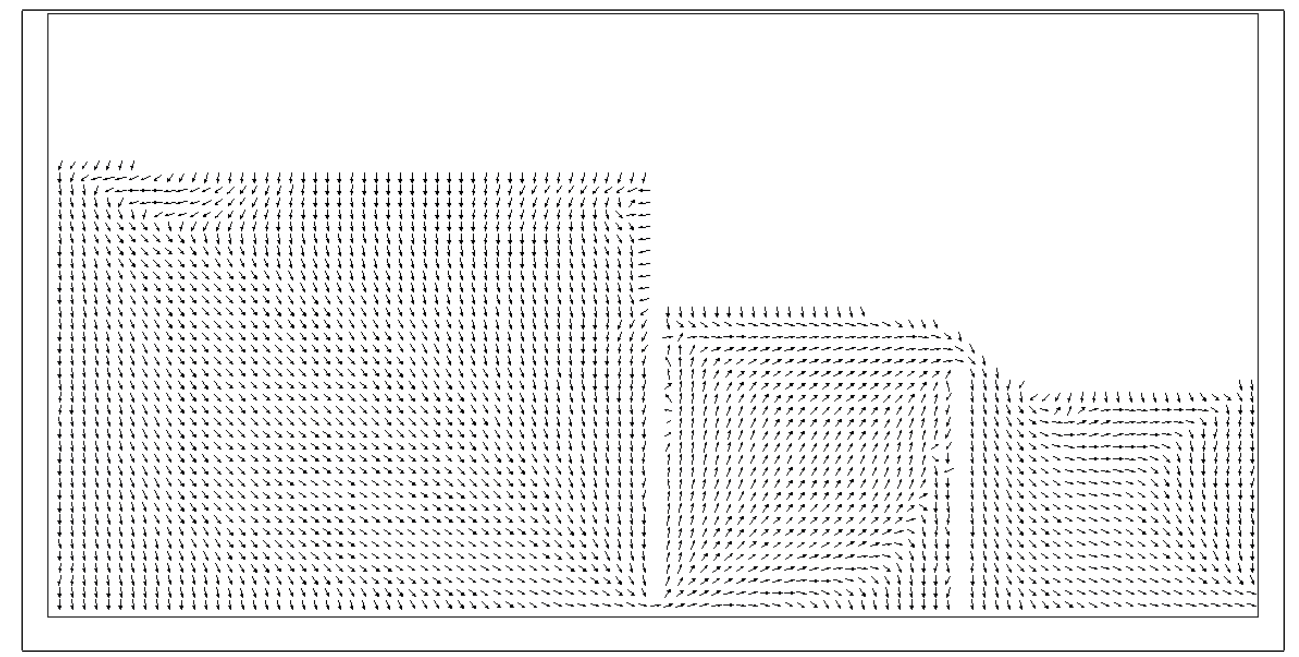

Figure 15: Velocity field for the draining LB simulation. The arrows represent the average speed in a $4 \times 4$ block of cells.

Note that the standard gate equation is derived from the Bernoulli law in a steady-state situation. We may thus expect a departure from the standard gate equation in the draining experiment, as the stationary hypothesis is no longer valid.

Of course, a first principle derivation would be interesting but, so far, we have not been able to proposed a clear cut theoretical explanation of this observed discrepancy with the standard gate equation. Note also that it is known that the gate coefficients may depend on the hydraulic variables [Lozano et al., 2009], showing that the standard gate equation has to be considered with care. Therefore, the discrimination is a difficult task. 


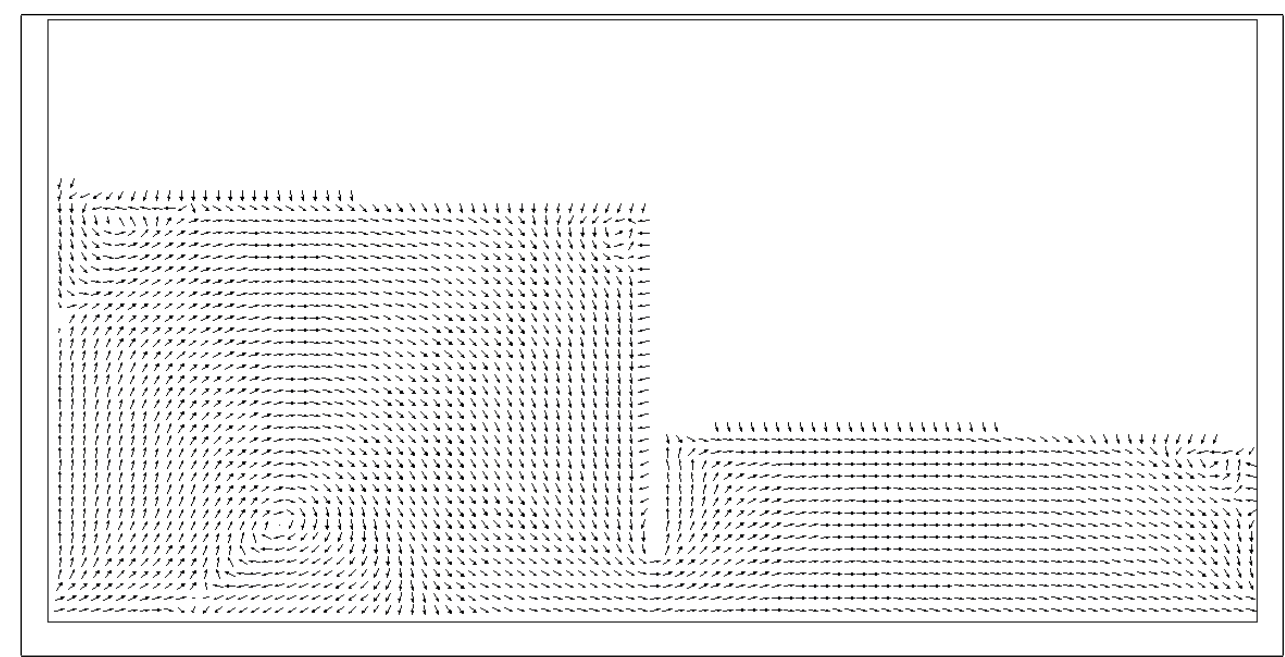

Figure 16: Velocity field for the stationary LB simulation. The arrows represent the average speed in a $4 \times 4$ block of cells. The Reynods number for the opening is around unity. The flow is not turbulent but recirculation zone are clearly visible and some quantitative similarity with the flow pattern of [Lozano et al., 2009] are observed. 


\section{Conclusion}

In this paper, we discussed a two-fluids water-air LB model with gravity forces producing a hydrostatic pressure field in the water component. In [Marcou et al., 2007], we have shown that this model also reproduces some important features of the shallow water equations, for instance the correct non-uniform water level in a slanted canal with friction forces. Although the equivalence with classical models is important, the relevance of this approach is more clearly stated here since we study the water dynamics around a gate, a situation in which, clearly, the classical use of the SaintVenant equations (in which the vertical component of the velocity field is not considered) do not apply.

We have shown that the LB model retrieves by its own the global lumpedparameter behavior of the classical gate equation from the local 2D description of the water dynamics in each lattice points. A still more convincing proof of the relevance of this model has been established in the emptying experiment where the classical gate equation does not explain the experimental results and where a linear gate equation fit the data much better. In this case the LB model provides results which perfectly agree with the experimental data.

Therefore, the LB model proves to be a computationally interesting alternative to classical discretization schemes applied to the free-surface NavierStokes equations. The LB dynamics is stated directly as a discrete collision- 
propagation model for idealized mesoscopic fluid particles and provides results which match the experimental ones in a satisfying way. Note also that the scheme is local and does not require any global step (such as the solving of a Poisson equation).

Moreover, as it will be shown in a forthcoming publication, only simple adaptations are sufficient to introduce algae or sediments populations in the LB model. The description of sedimentation, transport and erosion for "solid" point particles can be done by local and rather intuitive transition rules, as shown in [Dupuis and Chopard, 2002]. Preliminary simulations show moving sediments profiles in qualitative agreement with those observed experimentally in the micro-canal.

Another potential interest of our approach is to include water evaporation. It can be handled easily with the LB model since it is basically a water-air model in which we can adapt the water-air interaction forces to obtain a natural description of the evaporation process.

Among the future prospects of this work we intend to focus on two aspects.

The first direction concerns the multi-scale coupling of this model with classical shallow water models where only (1D or $2 \mathrm{D})$ horizontal components of the velocity field are represented. Such "horizontal" model could be described with discretization of the classical Saint-Venant equations, or with "one-fluid" LB model which already exists [Zhou, 2004]. Multi-scale 
coupling in such a sense is indeed a prerequisite to handle control problems for large scale and complex irrigation systems where it is not possible to describe the whole system at the detailed scale.

The second direction is concerned with special control problems which could need such 2D vertical and numerically effective models. The first foreseen problem is the cleaning or control of sediments in water reaches, specially around the gates. The control of algae growth, transport and sedimentation could be also of some interest. We obviously will greatly acknowledge any suggestion for additional problems where this modelling methodology could be useful as well.

We thank the Swiss National Science Foundation for financial support and the reviewers for their many corrections and useful suggestions which greatly improved the quality of this paper.

\section{References}

S. Ansumali, I.V. Karlin, S. Arcidiacono, A. Abbas, and N.I. Prasianakis (2007). Hydrodynamics beyond Navier-Stokes: Exact solution to the Lattice Boltzmann hierarchy. Phys. Rev. Lett., 98.

L. Axner, J. Bernsdorf, T. Zeiser, P. Lammers, J. Linxweiler, and A. G. Hoekstra. Performance evaluation of a parallel sparse Lattice Boltzmann solver. Journal of Computational Physics, 227(10):4895-4911, 2008. 
P.D. Bates, S.N. Lane, and R. Ferguson (2005). Computational fluid dynamics. Applications in Environmental Hydraulics. John Wiley and Sons, Ltd.

J.-P. Baume, J. Sau, and P.-O. Malaterre (1998). Modeling of irrigation channel dynamics for controller design. In IEEE International Conference on Systems, Man and Cybernetics (SMC'98), San Diego, California, pages $3856-3861$.

G. Besançon, J.F. Dulhoste, and D. Georges (2001). A nonlinear backstepping-like controller for a three-point collocation model of water flow dynamics. In Proceedings of IEEE Conference on Control Application CCA'2001, Mexico, Mexico.

P. Bhatnager, E. Gross, and M. Krook (1954). A model for collision process in gases. Phys. Rev., 94, 511.

R. Chartrand (2005). Numerical differentiation of noisy, nonsmooth data. Technical report, Los Alamos National Laboratory. http://math.lanl.gov/Research/Publications/Docs/chartrand-2007numerical.pdf.

S. Chaussinand (2003). Comparaison de lois de commande pour la gestion d'un micro-canal. Master's thesis, Grenoble Institute of Technology, Grenoble, France. 
S. Chen and G.D. Doolen (1998). Lattice Boltzmann method for fluid flows. Ann. Rev. Fluid Mech., 30:329-364.

B. Chopard (2009). Lectures on Lattice Boltzmann Methods for complex fluid flows, chapter High performance computing in Lattice Boltzmann models. Science4 Press.

B. Chopard and M. Droz (1998). Lattice-Gas Cellular Automata and Lattice Boltzmann Models: an Introduction. Cambridge University Press.

B. Chopard, P. Luthi, A. Masselot, and A. Dupuis (2002). Cellular automata and Lattice Boltzmann techniques: An approach to model and simulate complex systems. Advances in Complex Systems, 5-2:103-242.

V. T. Chow (1985). Open channel hydraulics. Mc Graw Hill, New York.

A.J. Clemmens, T. F. Kacerek, B. Grawitz, and W. Schuurmans (1998). Test case for canal control algorithms. J. Irrig. Drain. Eng., 124-1:23-30.

A.J. Clemmens, E. Bautista, B.T. Wahlin, and R.J. Strand (2005). Simulation of automatic canal control systems. J. Irrig. Drain. Eng., (131)4: $324-335$.

J.M. Coron, B. d'Andra Novel, and G. Bastin (1999). A Lyapunov approach to control irrigation canals modeled by saint-venant equations. In Proc. of the European Control Conference ECC'99, Karlsruhe, Germany, pages $3856-3861$. 
J. A. Cunge, Jr. F. M. Holloy, and A. Verwey (1980). Practical aspects of computational river hydraulics. Oitman, London.

S. Donath, K. Iglberger, G. Wellein, T. Zeiser, A. Nitsure, and U. Rude (2008). Performance comparison of different parallel Lattice Boltzmann implementations on multicore multisocket systems. Int. J. Comput. Sci. Eng., 4(1):3-11. ISSN 1742-7185. doi: http://dx.doi.org/10.1504/IJCSE.2008.021107.

J.F. Dulhoste, G. Besançon, and D. Georges (2001). Nonlinear control of water flow dynamics by input-output linearization based on collocation model. In Proceedings of the European Control Conference ECC'2001, Porto, Portugal.

A. Dupuis and B. Chopard (2002). Lattice gas modeling of scour formation under submarine pipelines. Journal of Mathematical Physics, 178(1):161 $-174$.

S. Geller, M. Krafczyk, J. Tolke, S. Turek, and J. Hron (2006). Benchmark computations based on Lattice-Boltzmann, finite element and finite volume methods for laminar flows. Computers $\&$ Fluids, 35(8-9):888-897.

I. Ginzburg (2005). Equilibrium-type and link-type Lattice Boltzmann models for generic advection and anisotropic-dispersion equation. Advances in Water Resources Pages, 28-11:1171-1195. 
I. Ginzburg (2007). Lattice Boltzmann modeling with discontinuous collision components: Hydrodynamic and Advection-Diffusion Equations. J. Stat. Phys., 126-1:157-206.

I. Ginzburg and K. Steiner (2003). Lattice Boltzmann model for free-surface flow and its application to filling process in casting. J. Comput. Phys., 185(1):61-99. ISSN 0021-9991. doi: http://dx.doi.org/10.1016/S00219991(02)00048-7.

W.H. Graf (1993). Hydraulique fluviale, Collection traité de genie civil. Presses polytechniques et universitaire romandes, Ecole polytechnique fédérale de Lausanne.

Z. Guo, C. Zheng, and B. Shi (2002). Discrete lattice effects on forcing terms in the Lattice Boltzmann method. Phys. Rev. E, 65.

B. Hamroun, L. Lefèvre, and E. Mendes (2006). Port-based modelling for open channel irrigation systems. Transactions on Fluid Mechanics, 1-12: 995-1009.

F.M. Henderson (1989). Open channel flow. Mc Millan Publishing Company, New york.

M. Junk, A. Klar, and L.-S. Luo (2005). Asymptotic analysis of the Lattice Boltzmann equation. Journal of Computational Physics, 210-2.

D. Kandhai (1999). Large Scale Lattice Boltzmann Simulation: Computa- 
tional Methods and Applications. PhD thesis, University of Amsterdam, Amsterdam, The Netherlands.

C. Koerner, M. Thies, T. Hoffmann, N. Thürey, and U. Rüde (2005). Lattice Boltzmann model for free surface flow for modeling foaming. J. Stat. Phys., 121(1/2):179-196.

J. Lätt (2007). Hydrodynamic Limit of Lattice Boltzmann equations. PhD thesis, University of Geneva, Switzerland. URL http://www . unige.ch/cyberdocuments/theses2007/LattJ/meta.html.

D. Lozano, L. Mateos, G. P. Merkley, and A. J. Clemmens (2009). Field calibration of submerged sluice gates in irrigation canals. J. of Irr. Drain. Eng., 135(6):763-772.

P.-O. Malaterre and J. Rodellar (1997). Multivariable predictive control of irrigation canals. design and evaluation on a 2-pool model. In International Workshop on the Regulation of Irrigation Canals : State of the Art of Research and Applications, RIC97, Marrakech, Morocco, pages 230238.

P.-O. Malaterre, D.C Rogers, and J. Schuurmans (1998). Classification of canal control algorithms. J. Irrig. Drain. Eng., 124-1:3-10.

O. Marcou, S. El Yacoubi, and B. Chopard (2006). A bi-fluid Lattice Boltz- 
mann model for water flow in an irrigation channel. In ACRI 2006 Proceedings., pages 373-382. Springer.

O. Marcou, B. Chopard, and S. El Yacoubi (2007). Modeling of irrigation canals: a comparative study. Int. J. Mod. Phys. C, 18 (4):739-748.

N. S. Martys and H. Chen (1996). Simulation of multi-components fluids in complex three-dimensional geometries by the Lattice Boltzmann method. Phys. Review E, 53(1).

W.A. Miller and J.A. Cunge (1975). Unsteady Flow in Open Channels, chapter Simplified equations of unsteady flows. Water Resources Publications, Fort Collins.

H. Ouarit, L. Lefèvre, and D. Georges (2003). Robust optimal control of onereach open-channels. In Proc. of European Control Conference ECC'2003, Cambridge, United Kingdom.

V.T. Pham (2009). Modélisation et commande des systèmes non-linéaire à paramètres distribués par la méthode de Boltzmann sur réseau: application aux canaux d'irrigation. Master's thesis, Grenoble Institute of Technology, Grenoble, France.

V.T. Pham, B. Chopard, L. Lefèvre, and E. Mendes (2010). Study of the 1d Lattice Boltzmann shallow water equation and its coupling to build a canal network. J. Comp. Phys.. Submitted. 
S. Sawadogo, P.O. Malaterre, and P. Kosuth (1995). Multivariable optimal control for on-demand operation of irrigation canals. International Journal of Systems Science, 26-1:161-178.

X. Shan and H. Chen (1993). Lattice Boltzmann model for simulating flows with multiple phases and components. Phys. Review E, 47, no 3.

X. Shan and H. Chen (1994). Simulation of nonideal gases and liquid-gas phase transitions by the Lattice Boltzmann equation. Phys. Review, 49, no 4 .

P.K. Stansby and J.G. Zhou (1998). Shallow-water flow solver with nonhydrostatic pressure: $2 \mathrm{~d}$ vertical plane problems. Int. J. Numer. Meth. Fluids, 28:161-174.

S. Succi (2001). The Lattice Boltzmann Equation, For Fluid Dynamics and Beyond. Oxford University Press.

M.C. Sukop and D.T. Thorne (2005). Lattice Boltzmann Modeling: an Introduction for Geoscientists and Engineers. Springer.

N. Thürey and U. Rüde (2009). Stable free surface flows with the Lattice Boltzmann Method on adaptively coarsened grids. Computing and Visualization in Science, 12 (5).

D. A. Wolf-Gladrow (2000). Cellular Automata Modeling of Physical Systems. Lecture Notes in Mathematics. Springer. 
X. Qing Xing, D. Lee Butler, and C. Yang (2007). Lattice Boltzmann-based single-phase method for free surface tracking of droplet motions. Int. J. Numer. Meth. Fluids, 53:333-351. DOI: 10.1002/fld.1282.

J.G. Zhou (2004). Lattice Boltzmann Methods for shallow water flows. Springer. 


\section{Tables}

Table 1: : Parameter values.

\begin{tabular}{|c|c|}
\hline$G_{R B}$ & -80.0 \\
$\Delta t$ & 0.00015 \\
$\Delta x$ & 0.0003 \\
$g$ & 10.0 \\
$\tau_{R}$ & 1.0 \\
$\tau_{B}$ & 2.0 \\
\hline
\end{tabular}

Table 2: Micro-channel parameters used for experiments

\begin{tabular}{|c||c|}
\hline length $L$ & 7 meter \\
\hline width $B$ & 0.1 meter \\
\hline slope $I$ & $1.6 \times 10^{-3}$ \\
\hline Manning-Strickler coefficient $K$ & 97 \\
\hline upstream gate parameter $\alpha_{1}$ & 0.66 \\
\hline downstream gate parameter $\alpha_{2}$ & 0.73 \\
\hline downstream outfall height $\left(H_{d e v}\right)$ & 0.05 meter \\
\hline
\end{tabular}

